\title{
Article \\ Study on the Relationship between Thermal Comfort and Learning Efficiency of Different Classroom-Types in Transitional Seasons in the Hot Summer and Cold Winter Zone of China
}

\author{
Haiqiang Liu ${ }^{1}$, Xidong Ma ${ }^{2, *}{ }^{\mathbb{D}}$, Zhihao Zhang ${ }^{1}$, Xiaoling Cheng ${ }^{1}$, Yanmi Chen ${ }^{3}$ and Shoichi Kojima ${ }^{4}$ \\ 1 School of Civil Engineering and Architecture, Zhejiang Sci-Tech University, NO.928 2nd Street, Qiantang \\ District, Hangzhou 310018, China; liu.haiqiang@zstu.edu.cn (H.L.); 2017331210017@mails.zstu.edu.cn (Z.Z.); \\ 2018337261004@mails.zstu.edu.cn (X.C.) \\ 2 School of Architecture, Tianjin University, NO.92 Weijin Road, Nankai District, Tianjin 300072, China \\ 3 School of Economics and Business Management, Zhejiang Sci-Tech University, NO.928 2nd Street, \\ Qiantang District, Hangzhou 310018, China; 2018333503069@mails.zstu.edu.cn \\ 4 Department of Civil Engineering and Architecture, Graduate School of Science and Engineering, \\ Saga University, Saga 840-8502, Japan; shokjm@cc.saga-u.ac.jp \\ * Correspondence: mxd2813@tju.edu.cn; Tel.: +86-188-1277-5772
}

Citation: Liu, H.; Ma, X.; Zhang, Z.; Cheng, X.; Chen, Y.; Kojima, S. Study on the Relationship between Thermal Comfort and Learning Efficiency of Different Classroom-Types in Transitional Seasons in the Hot Summer and Cold Winter Zone of China. Energies 2021, 14, 6338. https://doi.org/10.3390/en14196338

Academic Editor: Korjenic Azra

Received: 28 July 2021

Accepted: 30 September 2021

Published: 4 October 2021

Publisher's Note: MDPI stays neutral with regard to jurisdictional claims in published maps and institutional affiliations.

Copyright: (c) 2021 by the authors. Licensee MDPI, Basel, Switzerland. This article is an open access article distributed under the terms and conditions of the Creative Commons Attribution (CC BY) license (https:/ / creativecommons.org/licenses/by/ $4.0 /)$.

\begin{abstract}
The physical environment of classrooms has a strong relationship with student learning performance and health. Since the outbreak of COVID-19 in 2019, almost all universities have begun implementing closed instructional management, which has forced students to spend a much longer amount of time inside the classroom. This has also led to an increasing problem of thermal comfort in classroom indoor environments. In this paper, classrooms evolved from three dominant teaching modes at Zhejiang Sci-Tech University (ZSTU), located in the Hot Summer and Cold Winter (HSCW) zone of China, were selected as experimental spaces. Meanwhile, 12 learning groups with 60 students (30 of each sex) were selected as the tested samples. The relationship between thermal comfort and learning efficiency of the tested students was established through thermal comfort questionnaires and learning efficiency tests under the typical natural conditions in transition seasons. Based on this, improvement strategies were proposed for the current state of the classroom environment, providing a database for optimizing the environmental conditions of university classrooms in HSCW zone on the basis of improving students' learning efficiency.
\end{abstract}

Keywords: learning efficiency; thermal comfort; natural environment of different classroom-types; influence relationship

\section{Introduction}

With students spending approximately one-third of their day in the classroom, the physical environment of the classroom has a close relationship to students' learning and health [1]. Therefore, maintaining an appropriate classroom indoor environment is essential for the comfort and health of learning. In addition, with the outbreak of COVID-19, a large number of universities had to be forced into closed teaching management, which has extended the time that college students spent in the classroom. It is in this context that the quality of the university classroom environment is beginning to receive widespread attention. What is more, the impact on the classroom environment on student health is of even greater concern. However, the impact of the classroom environment on students' learning performance also deserves attention and research.

Currently, research on thermal comfort of indoor environments has been common, and many scholars have conducted relevant field and experimental studies, with establishing numerous evaluation models, design criteria, etc. [2-4]. With the advancement of technology, a number of codes and standards dealing with the basis of indoor thermal comfort 
assessment have also emerged internationally, such as ASHRAE Standard 55-2007 [4], ISO 7730-2005 [5], EN 16798-1 [6], and EN 16798-2 Standards [7], etc. There was also an international guideline that focuses on thermal comfort in teaching spaces, namely the REHVA Guidebook NO.13 [8]. Firstly, in the area of thermal comfort in classroom indoor environments, it has been found that there were differences in people's thermal comfort needs under different conditions. Some of these studies concluded that the thermoneutral temperature of the human body was closely related to age. Furthermore, by conducting a thermal comfort on both students and teachers in the classroom, it was found that children were more sensitive to higher temperatures than adults under the same conditions [9]. It was also concluded through a study of the current state of thermal comfort in elementary and secondary school classrooms that both values of neutral and desired temperatures were lower for students than for adults. In addition to age conditions, some studies have shown that students would feel more comfortable when the temperature of the classroom was slightly elevated [10-12]. In particular, students prefered warmer classroom environments during transitional seasons, indicating that the acceptable temperature for students was higher than the standard recommendation. However, maintaining adequate thermal comfort conditions in schools is difficult, especially in those hosted in rural historical buildings where it is impossible to install HVAC systems and provide mechanical cooling. Consequently, the metrics to be used could be different; for example, some scholars have proposed metrics such as ePMV and aPMV that are applicable under different experimental conditions [13-17]. Additionally, this series of past investigations were mostly performed through subjective satisfaction polls with little regard for the variability of the study sample, as well as the reduced number of those interviewed, thus making the findings limited.

As research related to the classroom indoor environment continues to intensify, in addition to the issue of students' thermal comfort, learning efficiency is also receiving increasing attention. At present, there are many studies in this field related to the influence of classroom indoor temperature and ventilation conditions on learning efficiency, and most of them use subjective evaluation and experimental methods [18-21]. Firstly, in terms of research on the effects of indoor temperature, it was found that for students' thinking skills, indoor temperature has a certain peak, i.e., people's thinking skills started to decrease when the temperature deviated from $26^{\circ} \mathrm{C}$ [18]. However, learning efficiency was not simply about thinking skills, and it also included concentration, memory, and so on. One way of assessing learning efficiency was also through the calculation of students' grades. Students' grades showed a significant increase when the indoor temperature was lowered from $25{ }^{\circ} \mathrm{C}$ to $20^{\circ} \mathrm{C}$, contrary to the single change in thinking skills [22]. Of course, in addition to the two abovementioned judging instruments, the use of neurobehavioural subtests such as number screening, graphic stacking and letter recognition were equally viable measures [23]. In terms of research into the effects of ventilation conditions, scholars have likewise made a great deal of research. With a high density of people and consequent poor ventilation conditions, the classroom was a crowded space, resulting in high indoor carbon dioxide concentrations, to the point where students' learning efficiency decreased [22,24]. When the indoor ventilation was unfavorable to students' learning efficiency, with the $\mathrm{CO}_{2}$ concentration being greater than 1000 ppm, people would take the initiative to open the windows for natural ventilation and improve indoor ventilation. In accordance with this, the above results were followed in this study. That was, the effect of $\mathrm{CO}_{2}$ concentration on the experiment was not taken into account while ensuring normal ventilation conditions in the room.

In parallel to studies on both indoor temperature and carbon dioxide concentration, a large number of scholars have also set out to investigate the mechanisms by which indoor lighting and acoustic environments affected learning efficiency $[25,26]$. For example, students' learning efficiency decreased as the colour temperature and illumination values of light sources increased, meanwhile, lighting settings should take into account the effects on learning efficiency and visual fatigue [25]. Another example was that students' learning 
efficiency varied under different musical atmospheres, among which baroque music had a certain effect on students' memory [26].

To sum up, the classroom environment is closely related to students' thermal comfort and learning, while the high density of people and the unpredictable outdoor temperatures during the transitional seasons lead to poor indoor comfort. However, up to now, researches on classroom environments have mainly focused on thermal comfort and less on learning efficiency. Most research has been conducted on the relationship between thermal comfort and learning efficiency through subjective evaluations such as thermal perception votes and thermal satisfaction votes. However, as is known to all, the participants in the experiments often had differences in physical fitness, which led to some errors in the subjective evaluations. As such, the results obtained from the studies are unclear and further research is necessary. Meanwhile, most of the research experiments were conducted in simulations under artificially set classroom environmental conditions with ad hoc results, and there was little research on the effect of classroom environment on students' thermal comfort and learning efficiency under natural conditions. As a complement, most of these experiments focused on a single indoor environmental parameter variable. However, in general, a holistic approach to explore all aspects of thermal comfort of the Indoor Environmental Quality, including IAQ, visual and acoustic comfort, will be more comprehensive and objective, which creates the possibility for future studies of multisensorial interactions $[27,28]$. Therefore, in order to fill the gap in the above-mentioned research area, the focus of this study is on the relationship between changes in students' thermal comfort and their own learning efficiency caused by changes in indoor environmental conditions (combined various changes in classroom temperature, relative humidity, illumination, etc.), under typical natural conditions during the transition season. The aim is to analyze the correlation between the current state of indoor environmental conditions, thermal comfort and learning efficiency through thermal comfort questionnaires and learning efficiency tests, providing guidance for improving indoor thermal conditions. Based on a certain level of thermal comfort, this study provides students in this climate zone with indoor environmental conditions more suitable for learning.

\section{Methodology}

\subsection{Location Selection}

China is a vast country and its climatic conditions vary greatly from place to place. To be exact, the temperature in the north can be as low as $-20^{\circ} \mathrm{C}$ in winter, while it can remain at $10{ }^{\circ} \mathrm{C}$ in the southernmost regions. Such various climatic conditions affect some extent the regional character of energy consumption in buildings. The Chinese national code "Thermal Design Code for Civil Buildings" [29] defines five climatic zones in China based on the average temperature of the coldest and hottest months, as shown in Figure 1.

Compared to other climatic zones, Hot Summer and Cold Winter (HSCW) zone, selected in this study, have a different significance in terms of annual climate change for the following reasons:

- This zone covers more than half of provinces in China and is home to over $40 \%$ of population in China. However, the small size of the zone, only $20 \%$ of China's total area, results in a higher population density than in other zones.

- The economic conditions of this zone are at China's forefront and come at a higher level than others, which has attracted a large number of labor migrants. On this basis, the number of educated people has grown rapidly and is now close to 30 million in this zone, with a correspondingly larger number of schools, teaching spaces and other facilities than in others.

- The climatic conditions of this zone are exceptional, with average outdoor temperatures of $0-10{ }^{\circ} \mathrm{C}$ in the coldest months and $25-30{ }^{\circ} \mathrm{C}$ in the hottest months (Figure 2). The relative humidity throughout the year is $70-80 \%$ or even higher (Figure 3 ). In addition to the summer and winter conditions, during the transitional season, this zone is characterized by a large difference in daily temperature due to the frequent 
intersection of cold and warm air in the middle and lower reaches of the Yangtze River, which makes the weather unpredictable, with frequent thunderstorms and coastal gales.

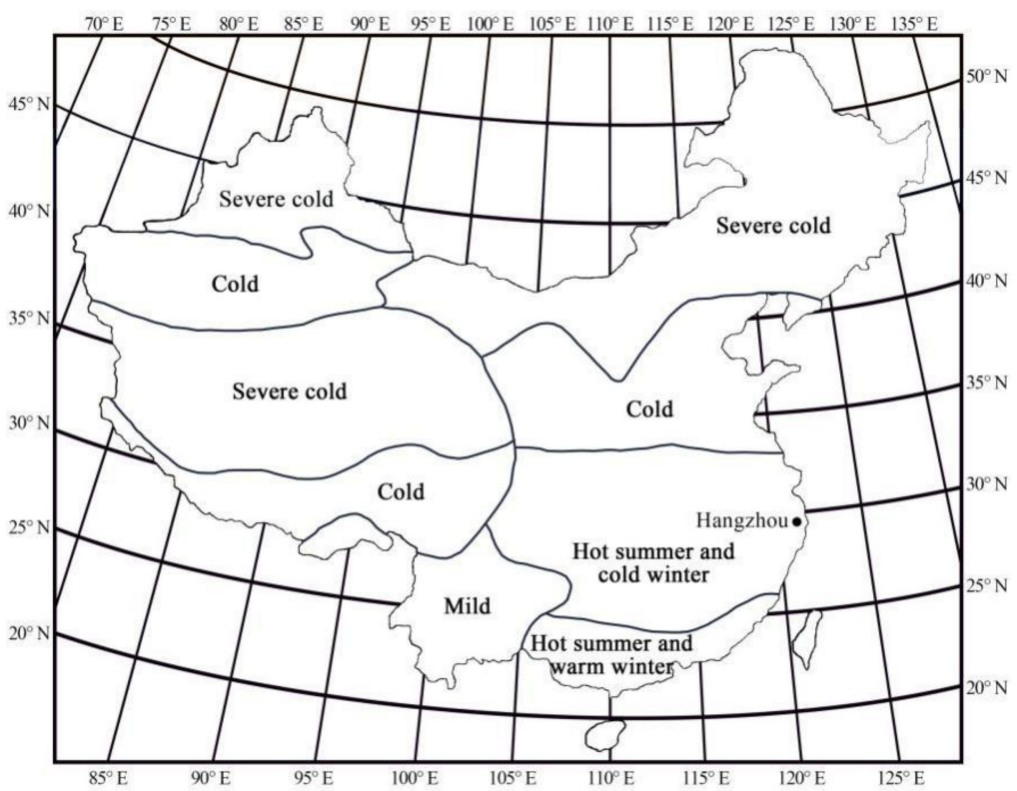

Figure 1. Layout of five climate zones in China and the survey city.

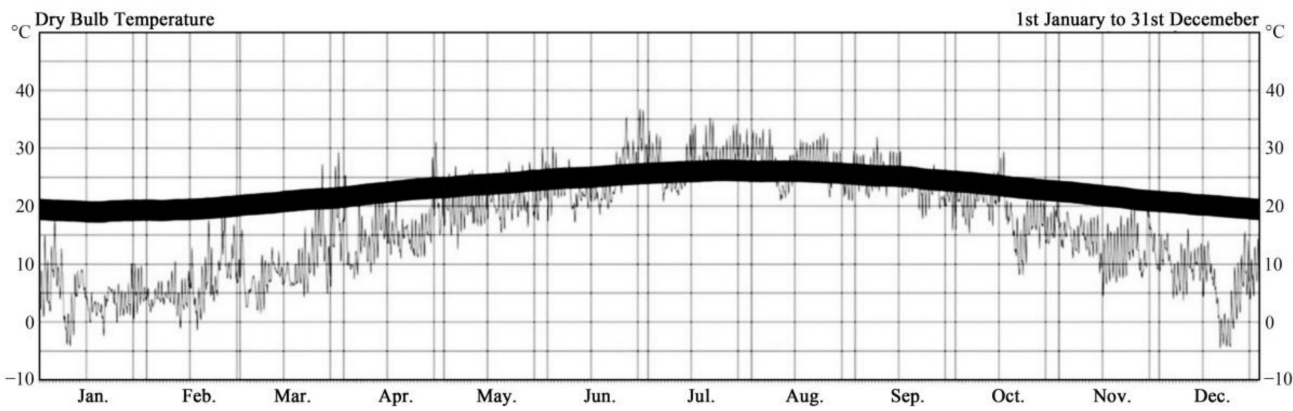

Figure 2. Daily dry bulb temperature profile in Hangzhou city.

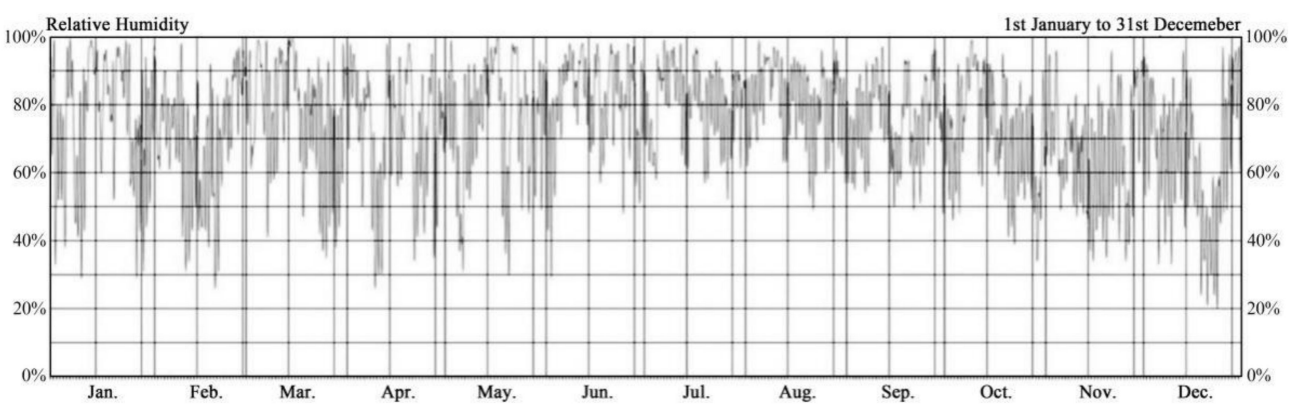

Figure 3. Daily relative humidity in the city of Hangzhou city.

A series of experience investigations of this study was carried out in Hangzhou, China, a city in the HSCW zone according to Chinese national standards. Hangzhou has a subtropical monsoon climate. Furthermore (Figure 4), the average minimum temperature is $6.3^{\circ} \mathrm{C}$ and the average maximum temperature is $25.5^{\circ} \mathrm{C}$ during the transition season (March to May), with minimum and maximum temperatures of $-1.9{ }^{\circ} \mathrm{C}$ and $35.6{ }^{\circ} \mathrm{C}$, respectively, and the average relative humidity of $76 \%$ to $81 \%$ [30]. 


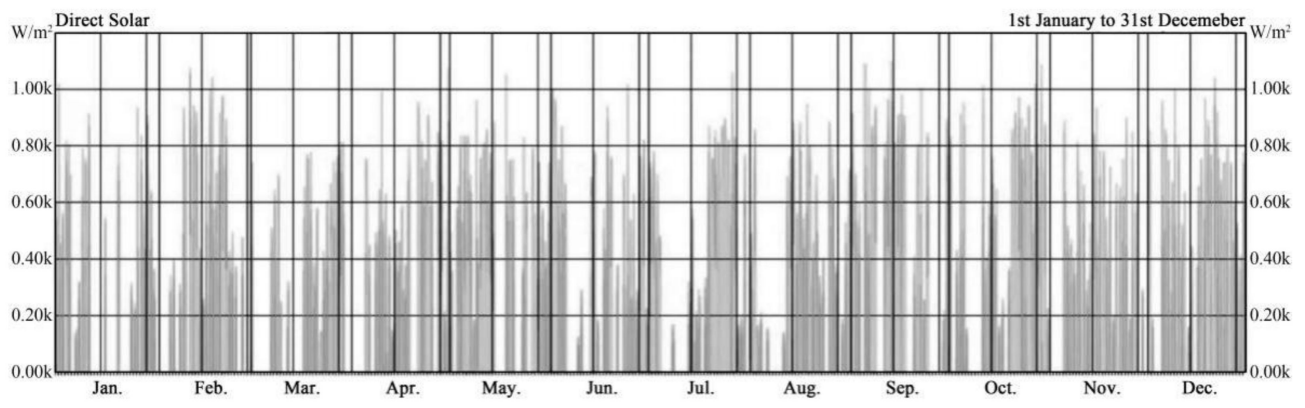

Figure 4. Daily direct sunlight in Hangzhou city.

\subsection{The Experiment Process}

All empirical surveys for this study were conducted in Zhejiang Sci-Tech University (ZSTU) in Hangzhou. Three months (March, April and May) during the spring of 2021, totaling 91 days, were used as the survey period. During these days, six sets of experiments were scheduled for each day, starting at 8:00 a.m. and ending at 20:30 p.m. Meanwhile, the detailed explanations and counseling were given to the students on the experimental procedures, and the determination of environmental parameters and the considerations related to the completion of subjective questionnaires before the experiments were conducted.

In addition, for selecting the subject students, the participating university students in this experiment were those who have lived in Hangzhou for a long period and were guaranteed to have similar information about their age, family background and so on. All students should ensure that they were in healthy and similar physical conditions without any diseases, such as cold, fever, or colour blindness. To ensure objectivity and accuracy, a range of variables needed to be avoided. For example, it was necessary to ensure that the number of male and female participants was the same to minimize the effect of gender on the results. Meanwhile, the number of students was also standardized to minimize the effect of the order of the learning efficiency tests, in line with the requirements of the Latin Hypercube Sampling (LHS) method [31]. By calculation, the working conditions were three in this experiment. To satisfy the LHS method, therefore, the number of students tested was at least six (or a multiple of six). Furthermore, to reduce the error caused by insufficient sample size, 60 students, 30 of each sex (the number of tested students remained the same, but there was a renewal of personnel, as shown in Table A1), were selected as participants in this experiment. In addition, for ensuring authenticity during the experiment, each student was asked to have a good routine before the experiment.

Four elements, namely adaptation time, environmental measurements, learning efficiency tests and subjective evaluation of thermal comfort, were included in this experiment. As illustrated in Figure 5, the duration of the entire investigation was approximately $70 \mathrm{~min}$. The first part was the environmental adaptation session, which took $30 \mathrm{~min}$. During this time, students could read books and do homework to enter the study state as quickly as possible, but had to remain quiet and ensure a calm state. Simultaneously, the basic environmental parameters of the classroom were measured using the relevant equipment (Test A) and this session lasted for the whole day. Afterward, the students were given a learning efficiency test (Test B) for approximately $15 \mathrm{~min}$, which was described in detail below. Finally, subject students would then complete a subjective evaluation of indoor thermal comfort (Test C) in the last 5 min of the experiment. These were all that need to be completed for each group of experiments. 


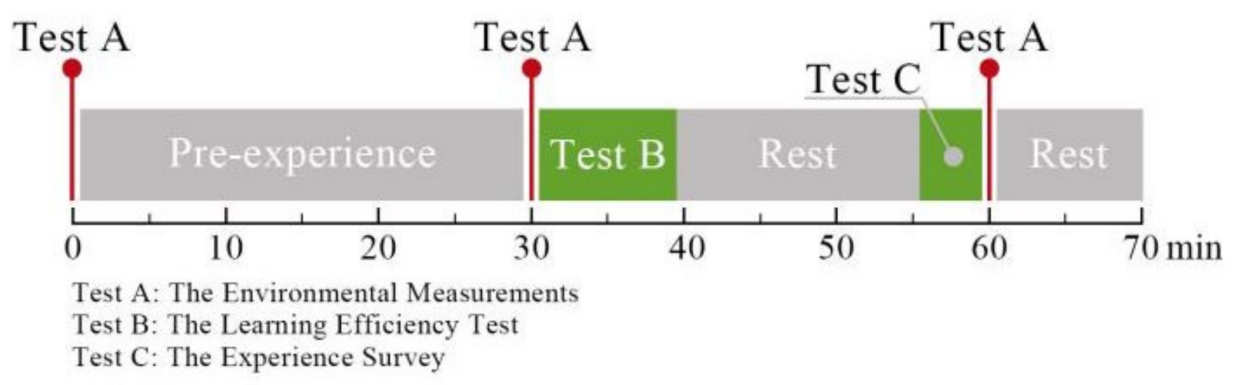

Figure 5. Schedule of the experiment process.

\subsection{The Environmental Measurements}

A number of related studies have counted the basic characteristics of Chinese university classrooms, including function, size, capacity, and so on [32-34]. The results show that the classification of university classrooms is inextricably linked to their teaching modes. The University curriculum consists of three different teaching modes: small classes, medium-sized workshops, and group lectures, which directly affect the size and capacity of the classroom as each mode of teaching targets a different number of students. Therefore, it is evident that the different teaching models in the university have created different types of classrooms, which can be divided into small, medium, and large classrooms, as shown in Table 1. In this study, three classrooms of different sizes were selected for experimentation in the same educational building in ZSTU.

Table 1. Relationship between the curriculum types and the classroom types.

\begin{tabular}{ccc}
\hline Curriculum Type & Capacity & Classroom Type \\
\hline Small Class & about 20 students with the area of about $35 \mathrm{~m}^{2}$ & Small Classroom \\
Medium-sized Workshop & about 60 students with the area of about $90 \mathrm{~m}^{2}$ & Medium Classroom \\
Group Lecture & about 200 students with the area of around $360 \mathrm{~m}^{2}$ & Large Classroom \\
\hline
\end{tabular}

Finally, these three case models were selected as follows:

- CASE A: Small Classroom

- CASE B: Medium Classroom

- CASE C: Large Classroom

The layout of the three cases is shown in Figure 6, and the basic information is also shown in Table 2. As it can be seen, the three classrooms differ in floor areas, shape coefficients, and window-to-floor ratios, which are the main reasons for the difference in the indoor environment of the three classrooms. However, all three classrooms have the same envelope, as in the same educational building, what's mean that all are of brick construction with aluminum single pane windows. Further explanation, the small classroom can accommodate up to 25 people, the medium classroom can hold 80 people, while the large classroom can accommodate 180 people. All classrooms tested were under natural conditions, with no cooling or heating, no ventilation systems, and all windows closed. Further, all classrooms selected were located on the south side of the building, so the indoor environment reflected significant fluctuations directly through access to solar energy from the outdoors during the day.

In this experiment, the basic conditions of indoor and outdoor environments, including air temperature $(\mathrm{Ta})$, relative humidity $(\mathrm{RH})$, air velocity $(\mathrm{Va})$, and illuminance $(\mathrm{E})$, were measured and recorded every $15 \mathrm{~min}$ with the appropriate measuring equipment. Table 3 shows the type of equipment used to determine each environmental parameter. Figure 6 also presents the placement of the equipment in the three classrooms and the specific layout of the experimental environment, with all equipment fixed at a height of $1.1 \mathrm{~m}$ above 
the floor in accordance with ISO 7726 [35]. All equipment was calibrated periodically in accordance with the relevant instructions for use, before conducting the experiments.

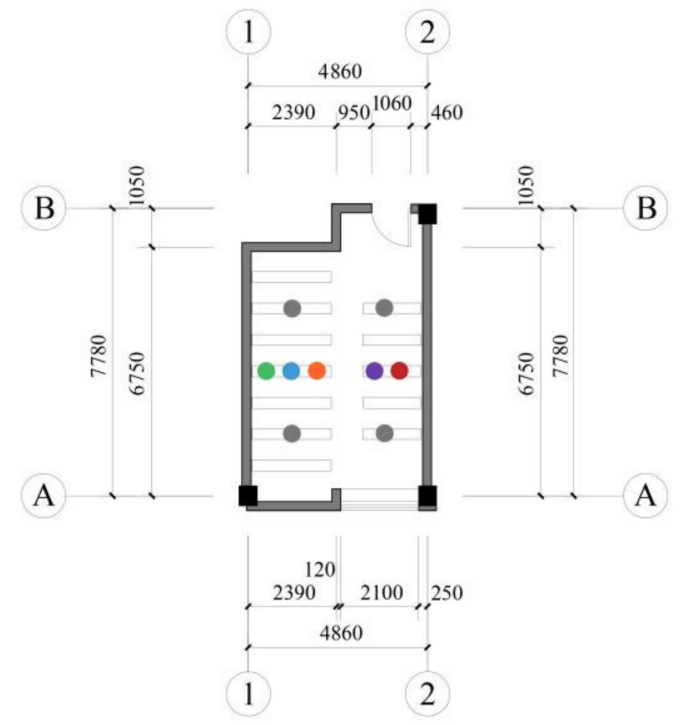

CASE A: Small Classroom

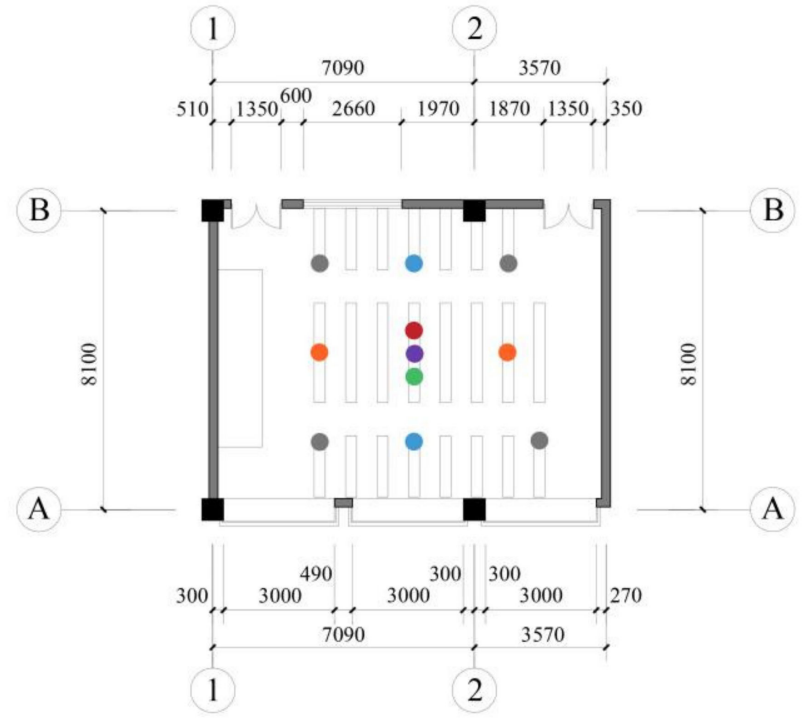

CASE B: Medium Classroom

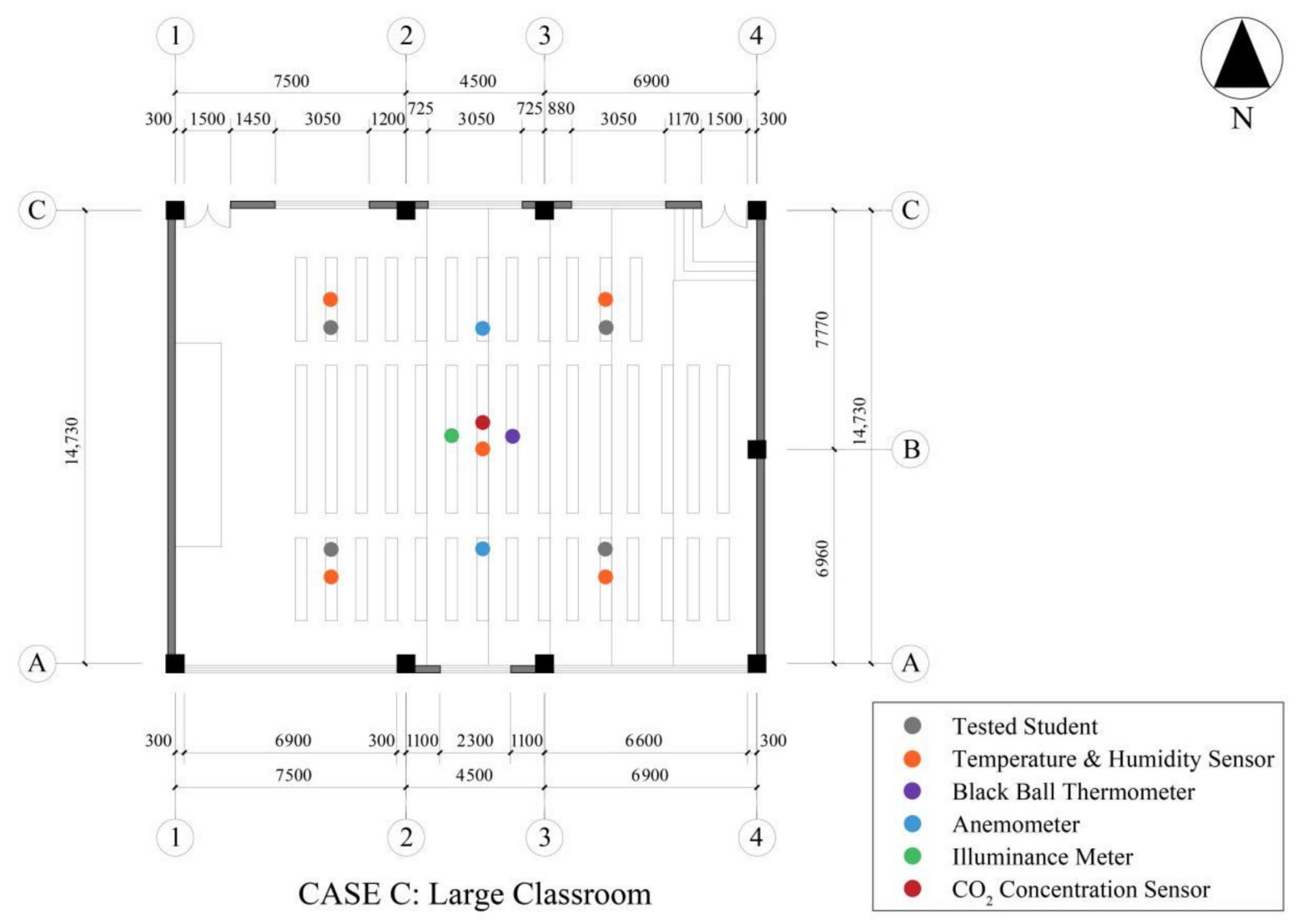

Figure 6. Layout of the case A, B, and C. 
Table 2. Basic information of the case A, B, and C.

\begin{tabular}{cccc}
\hline Classroom Type & Small Classroom & Medium Classroom & Large Classroom \\
\hline Case & A & B & C \\
Area $\left(\mathrm{m}^{2}\right)$ & 33.70 & 90.74 & 279.19 \\
Shape Coefficient & 1.08 & 0.64 & 0.57 \\
Window-to-floor Ratio & 0.11 & 0.12 & 0.18 \\
Orientation & South-facing & South-facing & South-facing \\
Floor Level & 2nd floor & 2nd floor & 2nd floor \\
Wall Alternatives & Concrete Wall Insulated & Concrete Wall Insulated & Concrete Wall Insulated \\
U-value & Semi-hard Mineral Wool & Semi-hard Mineral Wool & Semi-hard Mineral Wool \\
Board Outside & Board Outside & Board Outside \\
& 1.09 & 1.09 & 1.09
\end{tabular}

Table 3. Basic information of the experimental equipment.

\begin{tabular}{ccccc}
\hline Parameters & Instrument/Sensor & Time Interval & Range & Accuracy \\
\hline Air Temperature & $\begin{array}{c}\text { T-type Thermocouple-GRAPHTEC } \\
\text { Midi Logger GL220 }\end{array}$ & $1 \mathrm{~min}$ & $0-50{ }^{\circ} \mathrm{C}$ & $\pm 0.3^{\circ} \mathrm{C}$ \\
Globe Temperature & AZ Handheld WBGT Tester 8778 & $15-25 \mathrm{~min}$ & $0-80{ }^{\circ} \mathrm{C}$ & Indoor: $\pm 1{ }^{\circ} \mathrm{C}\left(15-40{ }^{\circ} \mathrm{C}\right)$ \\
Relative Humidity & Thermo Recorder TR-72Ui & $1 \mathrm{~min}$ & $10-95 \% \mathrm{RH}$ & $\pm 1.5{ }^{\circ} \mathrm{C}\left(15-40^{\circ} \mathrm{C}\right)$ \\
Air Velocity & Hotline Anemograph Testo 425 & $10 \mathrm{sec}$ & $0-20 \mathrm{~m} / \mathrm{s}$ & $\pm 0.03 \mathrm{~m} / \mathrm{s}+5 \%$ \\
Illuminance & Light Sensor RS485 & $0.1 \mathrm{sec}$ & $0-200 \mathrm{k} \mathrm{lux}$ & $\pm 7 \%$ \\
$\mathrm{CO}_{2}$ Concentration & Handheld CO 2 Detector 7755 & $30 \mathrm{sec}$ & $0-9999 \mathrm{ppm}$ & $\pm 50 \mathrm{ppm}+5 \%$ \\
\hline
\end{tabular}

\subsection{The Experience Survey}

The experiment conducted every day was consisted of six small tests, in each of which a thermal experience survey was administered, obtaining a total of six thermal experience evaluations per day. During the adaptation session, the experience reports were distributed to each student, and a short but detailed guide was also given to ensure that the students were aware of the academic terms used in the questionnaire.

The experience questionnaire was divided into three sections, namely Basic Information, Thermal Sense Vote (TSV) and Thermal Approving Vote (TAV). Firstly, basic information included information such as gender, age and person number of the subject students. All subject students were also told to choose similar types of clothing (a long sleeve, a pair of trousers and a coat) before the experiment started, to ensure that the students were dressed as uniformly as possible in terms of insulation. Lastly, and most importantly, TSV and TAV were the two main parameters used to assess the thermal experience of subject students. Based on the seven-point ASHRAE criteria [4], Table 4 shows that TSV was assessed on a seven-point scale from -3 (Cold) to 3 (Hot), and TAV was assessed from -3 (Very Dissatisfied) to 3 (Very Satisfied) too.

Table 4. Thermal sensation scale of TSV, TAV and PMV.

\begin{tabular}{cccc}
\hline Scale & TSV & TAV & PMV \\
\hline+3 & Hot & Very Satisfied & Hot \\
+2 & Warm & Satisfied & Warm \\
+1 & Slight Warm & Slightly Satisfied & Slight Warm \\
0 & Neutral & Acceptable & Neutral \\
-1 & Sightly Cool & Slightly Dissatisfied & Sightly Cool \\
-2 & Cool & Dissatisfied & Cool \\
-3 & Cold & Very Dissatisfied & Cold \\
\hline
\end{tabular}

The 60 tested students ( 30 males and 30 females) selected for the experiment were divided equally into groups of 20 in three tested classrooms. In each classroom, the 
20 tested students were further divided into four small groups of five, distributed in four directions in the classroom. Each group of five would generate an average of the thermal comfort votes (TSV and TAV), which was intended to indicate that there was also some variation in the thermal comfort in different orientations. Therefore, in each tested classroom, four average values of thermal comfort votes representing the four directions would be generated, for a total of 12 averages in the whole experiment.

The TSV and TAV mentioned above were the results of students' subjective thermal comfort evaluation. In this study, the PMV/PPD model, proposed by P.O. Fanger using the heat-balance equations and empirical studies on skin temperature, was chosen as a way to define comfort. As shown in Table 4, in this model, subjects were asked about their thermal sensations through a standard thermal comfort survey, and people's thermal sensations were classified on a seven-point scale from cold $(-3)$ to hot $(+3)$. During this experiment, Fanger's equations were used to calculate the PMV for a specific combination of air temperature, mean radiant temperature, relative humidity, air velocity, metabolic rate and basic clothing insulation in the tested classrooms [36,37]. Additionally, the PMV was a predictive value that would be compared with TSV and TAV in the later study.

In the process of calculating PMV, in addition to environmental parameters such as air temperature, there are also some parameters that are predicted based on the current situation, including the metabolic rate value and the basic clothing insulation value. According to the ASHRAE Standard 55 [4], for example, since the tables and chairs in the tested classrooms were all wooden, their thermal insulation could be ignored. Additionally, the tested students in the experiment were asked to wear similar clothes (a long sleeve, a pair of trousers and a coat) in advance, so it could be predicted that the basic clothing insulation (lcl) was about 0.75 clo [38]. The students were in a quiet sitting position for reading and writing during the experiment, so their metabolic rate (M) could be chosen to be 1.2 met [39]. Finally, the other environmental parameters corresponded to the measured data under different experimental conditions, and the PMV was calculated separately for different experimental conditions.

\subsection{The Learning Efficiency Test}

The learning efficiency test is a research for students' efficiency of learning, but there are relatively few relevant efficiency assessment tests in this field. In contrast, there are numerous assessment methods on productivity that are also brain-based. Therefore, a similar assessment method should be selected and modified in this experiment, on the premise that the two have a high degree of similarity. It is a neuro-behavioural test that is widely used to assess the productivity of office workers, which focuses on various neuro-behavioural abilities, including perception, memory, concentration, thinking and emotional control [40]. This method is not only short and suitable for testing students learning efficiency, but the overall test is composed of a series of mini-games in a simple format. So this method was used in this experiment.

For the specific content, this test was mainly based on the APCD (Attention, Perception, Comprehension and Deduction) method [23]. Through the comprehensive evaluation of these four aspects, a more objective representation of the students' learning efficiency was derived. Therefore, this test was based on the APCD method, which developed the assessment of learning efficiency in four dimensions, including Perceptual Ability, Memory Ability, Concentration Ability and Thinking Ability, as shown in Table 5. The relevant test games were also adapted and optimized. Table A2 in Appendix A shows all the test items used in this experiment and the corresponding methods of operation [41-47]. 
Table 5. Four dimensions and main test items of the APCD method.

\begin{tabular}{cc}
\hline Learning Efficiency Dimension & Test Item \\
\hline Perceptual Ability & Letter Search, Word Color Interference, Graphic Overlay, \\
Concentration Ability & and Stereo Vision \\
Memory Ability & Schulte Grid and Digital Filter \\
Thinking Ability & Visual Learning and Memory Scanning \\
\end{tabular}

Ultimately, the learning efficiency was quantified by counting the average correct proportion (ACP) and average reaction time (ART) of students completing each test item. For this part of the data sorting, the values with obvious errors should be removed before the statistical average can be processed next. As such, there were two key points to note in the statistical component of the data. On the one hand, it is not feasible to directly compare test results across items as students' performance varies from test to test. Thus, in this experiment, the ACP and ART must be normalized at first, and all data should be processed by the process expressed in Equation (1) for calculation [47].

$$
P_{i, j}(\%)=\frac{x_{i, j}}{\frac{1}{n} \sum_{j=1}^{n} x_{i, j}} \times 100
$$

where $x_{i, j}$ is an index of the $i$-th student under working condition $j$, and $n$ refers to the total number of working conditions.

On the other hand, one indicator alone may not give a very objective picture of either $\mathrm{ACP}$ or ART, as there may be cases where students got a high percentage correct but took a long time. Thus, a composite indicator needed to be introduced to represent the combined efficiency of each item, and Equation (2) shows the assessment indicator item combined ACP and ART, namely the combined Learning Performance (LP), which represented the combined impact of student correctness and response time [47]. To sum up, the three indicators mentioned above were all used as the basis for evaluating student learning efficiency in the later stage.

$$
L P=\left[A C P^{0.5} \times(1 / A R T)^{0.5}\right]^{2}
$$

\section{Data Analysis}

\subsection{Determination of Indoor and Outdoor Environmental Parameters}

In this experiment (from 1 March 2021 to 30 May 2021), the environmental parameters were mainly measured, including indoor temperature, outdoor temperature, relative humidity, central illuminance, air velocity and $\mathrm{CO}_{2}$ concentration. Furthermore, the 91 days were divided into three categories, i.e., sunny, rainy and cloudy, based on the principle of weather conditions, and the environmental parameters were calculated and plotted as average curves. Finally, as presented in Figures 7-9, by comparing the change curves, it was found that the change curves of these three days (March 21, March 27 and March 28) were the most similar to the mean curve, which indicated that they could be sufficiently representative of the whole transition season.

Figure 7 shows the indoor and outdoor temperature changes in the three classrooms under cloudy, rainy and sunny conditions, respectively. It can be found that the temperature in each classroom was various under different weather conditions. Additionally, regardless of the weather conditions, the indoor temperature of the large classroom was the lowest, while the indoor temperature of the small classroom was the highest, followed by the medium classroom. Over the day, the indoor temperature of the small classroom had the largest change, followed by the medium classroom, while the change of the large classroom was the smallest. 


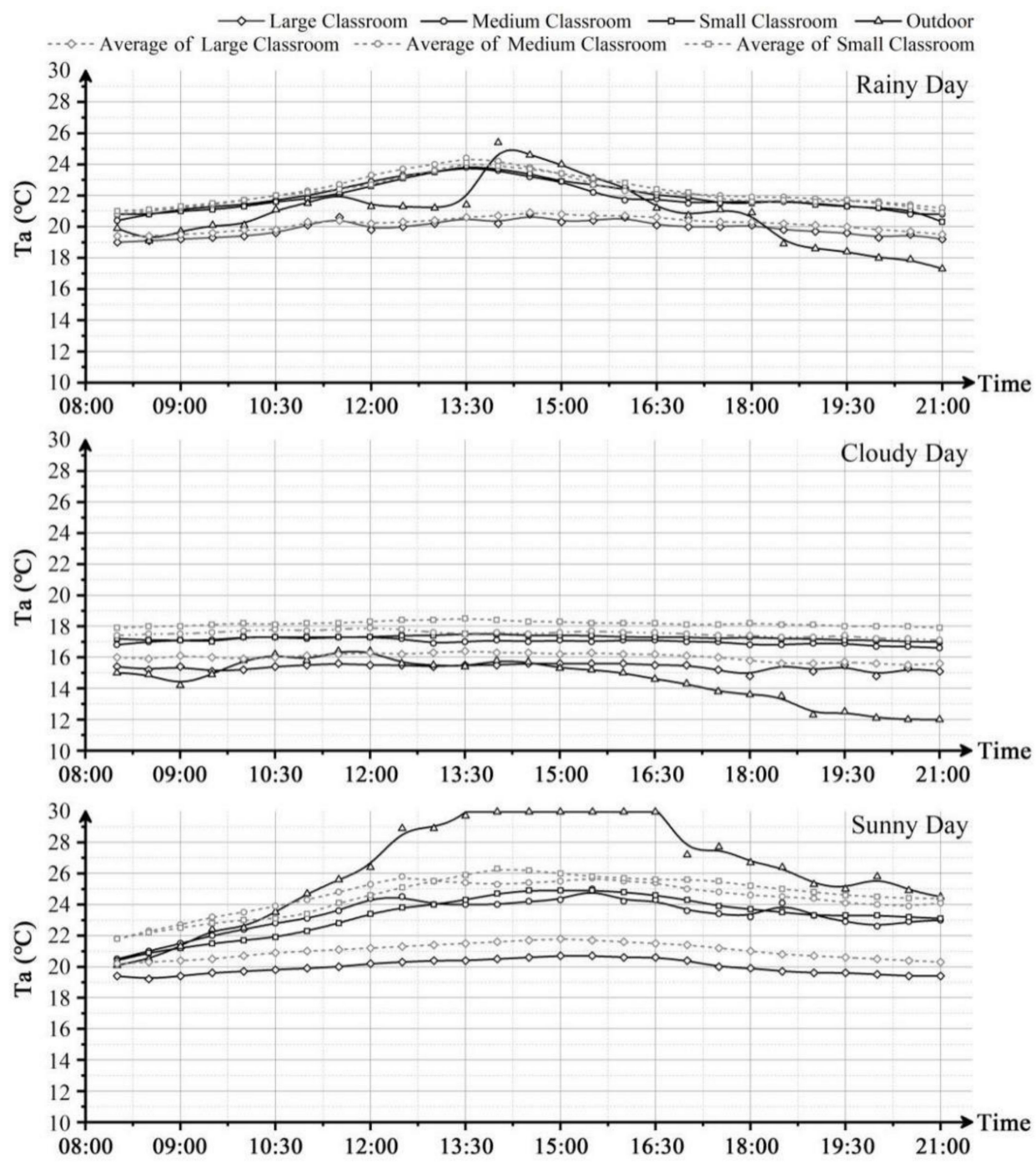

Figure 7. Temperature in the three classroom-types under different weather conditions throughout the day.

Figure 8 presents the relative humidity changes in the three classrooms under different weather conditions. Firstly, in general, the indoor relative humidity in rainy days was higher compared to other days, and the relative humidity varies less. Furthermore, in all three weather conditions, the relative humidity of large classroom was higher than the other two types, while the small classroom had the lowest relative humidity. Then, the indoor relative humidity in sunny days varied the most throughout the day, followed by cloudy days, and the least under rainy conditions. Finally, regardless of the weather conditions, the change in relative humidity in the small classroom was the most significant among the three classroom types. 


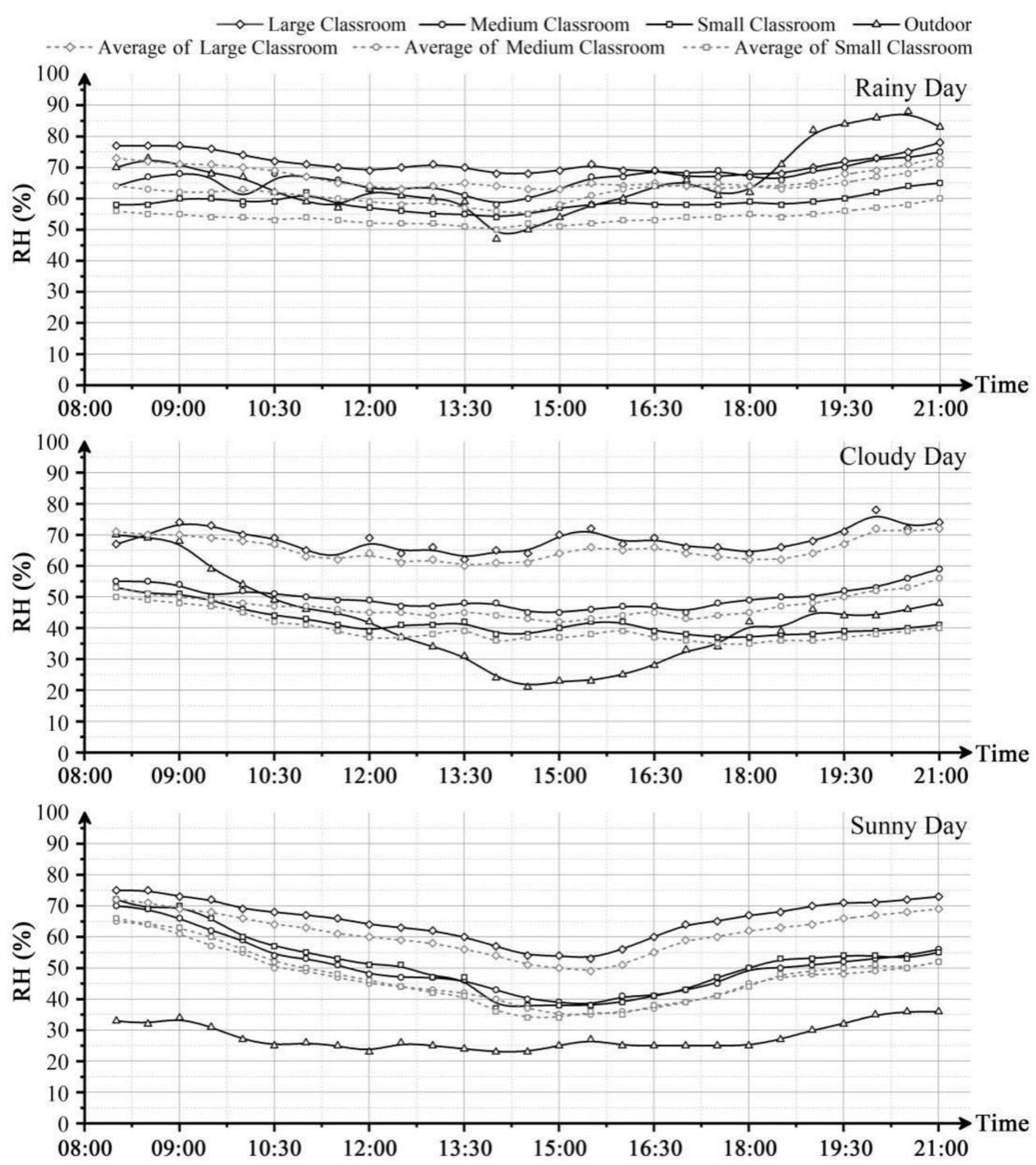

Figure 8. Humidity in the three classroom-types under different weather conditions throughout the day.

Figure 9 illustrates the changes of the central illuminance in the three classrooms under cloudy, rainy and sunny conditions, respectively. Firstly, it should be noted that the central illuminance values were measured under normal outdoor solar radiation (without direct solar radiation because of the distance between the measurement point and the external windows), and the values of the three classrooms showed significant differences. Secondly, the $\mathrm{E}$ values under the sunny condition were the highest of the three weather conditions. However, in the range of 12:00 to 13:30 under the cloudy condition, there was a short period of sunny sky, which led to the E values greater than 1000 lux in both the small and medium classrooms. In addition, since the measurement points in the small and medium classrooms were very close to the windows, while the large classroom's were much further, the $\mathrm{E}$ values in the large classroom were the lowest compared to the other two types. In general, although there was no direct solar radiation, the $\mathrm{E}$ values at all times met the relevant design standard "Hygienic Standard for Day Lighting and Artificial Lighting for Middle and Elementary School" (GB7793-2010) [48], with no significant impact on learning efficiency, due to the lights always being on and the white decorations in the classrooms. 

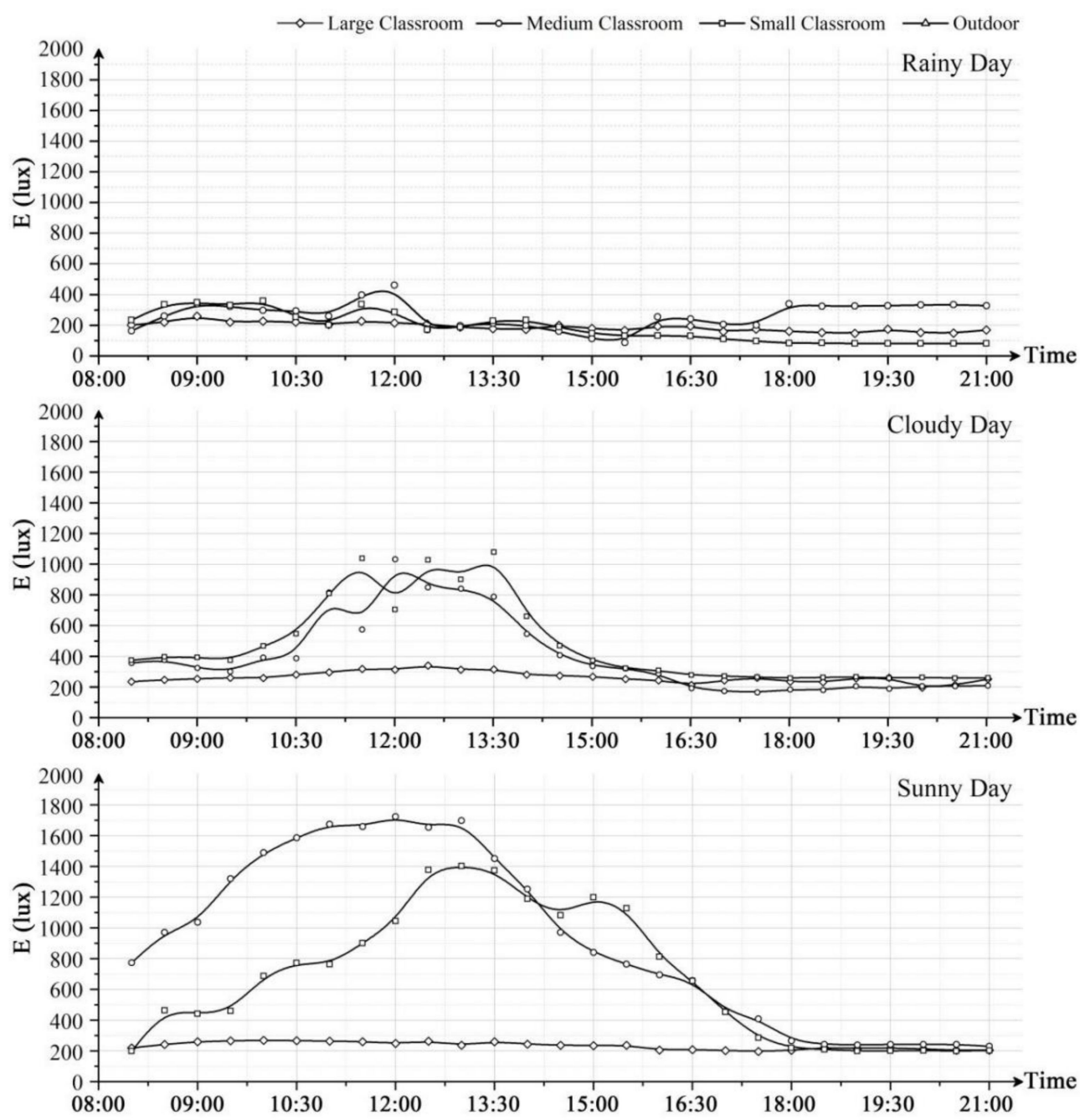

Figure 9. Central illuminance in the three classroom-types under different weather conditions throughout the day.

In accordance with the three environmental parameters above, the variation of air velocity and $\mathrm{CO}_{2}$ concentration were also measured in the experiment. However, the results showed that their variation were small regardless of the weather conditions, where the air velocity was stable at about $0.10 \mathrm{~m} / \mathrm{s}$, and the $\mathrm{CO}_{2}$ concentration was at a stable and comfortable level of about $400 \mathrm{ppm}$ [24]. What is more, the air temperature (Ta) and the globe temperature $(\mathrm{Tg})$ were all recorded together in this experiment. On this basis, the mean radiant temperature ( $\mathrm{Tr}$ ) and the operative temperature (To) values have been derived according to ISO 7726. However, since the temperatures were measured in center of the classrooms, where there was no direct solar radiation, and not exposed to air velocities greater than $0.10 \mathrm{~m} / \mathrm{s}$, it could be assumed that the values of $\operatorname{Tr}$ and To were replaced by Ta in the further calculation process, according to the ASHRAE Standard 55 [4] for determining the operative temperature.

\subsection{Data Analysis of Thermal Comfort Evaluation}

\subsubsection{TSV and TAV}

The results of the TSV and TAV of the tested students in the three classroom-types under different weather conditions throughout the day are shown in Figures 10 and 11. 


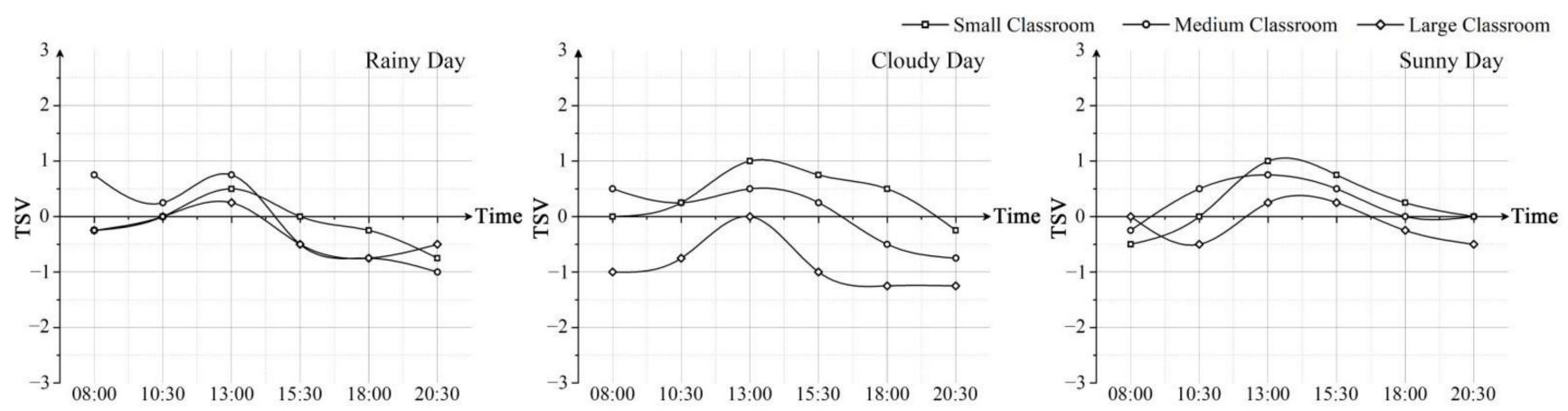

Figure 10. The TSV of the tested students in the three classroom-types under different weather conditions throughout the day.

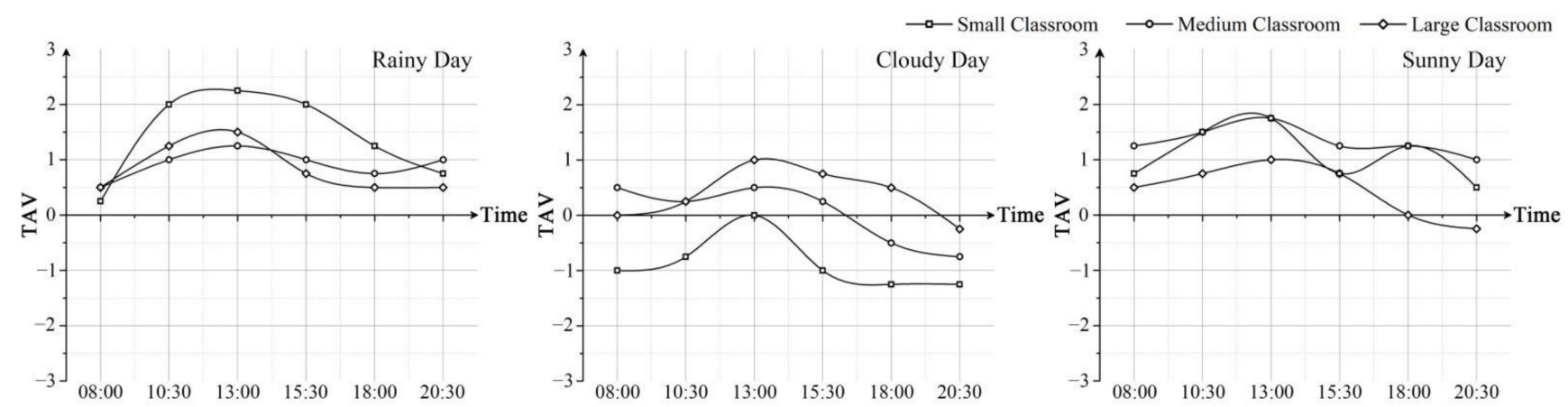

Figure 11. The TAV of the tested students in the three classroom-types under different weather conditions throughout the day.

From the analysis of environmental parameters above, it can be concluded that the temperature on sunny days was higher than on cloudy days and lowest on rainy days. As shown in Figure 10, the proportion of TSV as warm increased on sunny days when the temperature was higher. On rainy days with lower temperatures, the proportion of TSV as cooler grew relatively. However, under the same weather conditions, the TSV differed between classroom-types because of different environmental conditions. Moreover, regardless of the weather conditions, the TSV in all three classrooms showed the same trend throughout the day, with an increase followed by a decrease and the highest TSV value scoring at around 13:00.

As for the TAV, it can be discussed in terms of both different weather and classrooms. As presented in Figure 11, under different weather conditions, the TAV on sunny days with higher temperatures was relatively higher, but only around +1 . Simultaneously, the $\mathrm{TAV}$ on the rainy day was the lowest, with an average value of about -0.5 . Alternatively, under the same weather conditions, the TAV in different classrooms showed a similar trend throughout the day, increasing firstly and then decreasing, reaching the highest values at around 13:00. To sum up, during the transition season, most tested students were dissatisfied with the thermal environment in the lower temperature environments when compared to higher temperature environments.

As indicated in Figures 12 and 13, the relationship curves between TSV, TAV and temperature were plotted by combining the heat sensation voting and heat satisfaction voting data measured in three types of weather and in different classrooms. In detail, Figure 12 represents the variation of the TSV between -3 (Cold) and +3 (Hot) over the temperature range of $14^{\circ} \mathrm{C}$ to $25^{\circ} \mathrm{C}$ (the current situation in the transition season). Figure 13 shows that TAV varied between -3 (Very Dissatisfied) and +3 (Very Satisfied) over the temperature range of $14^{\circ} \mathrm{C}$ to $25^{\circ} \mathrm{C}$ (the current situation in the transition season). However, according to the ASHRAE Standard 55 [4], the thermal sensation is "neutral" at a temperature of approximately $26.5^{\circ} \mathrm{C}$, which is supposed to be a critical value. However, since this study 
was conducted during the transitional season, the measured temperatures ranged from $14{ }^{\circ} \mathrm{C}$ to $25^{\circ} \mathrm{C}$; as the temperature increased, the TAV increased and did not show a change in the transition. In summary, it can be shown that the tested students preferred a warmer environment during the transition season.

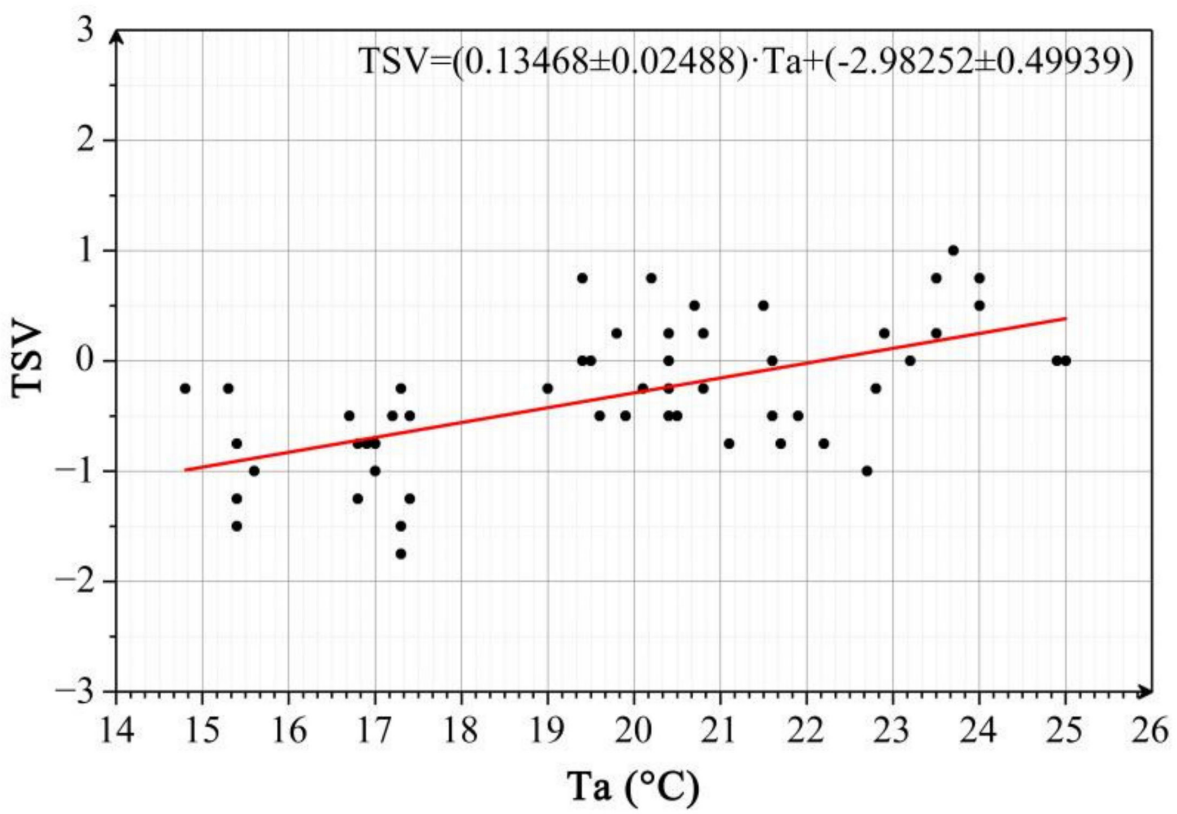

Figure 12. The quantitative relationship between TSV and Ta.

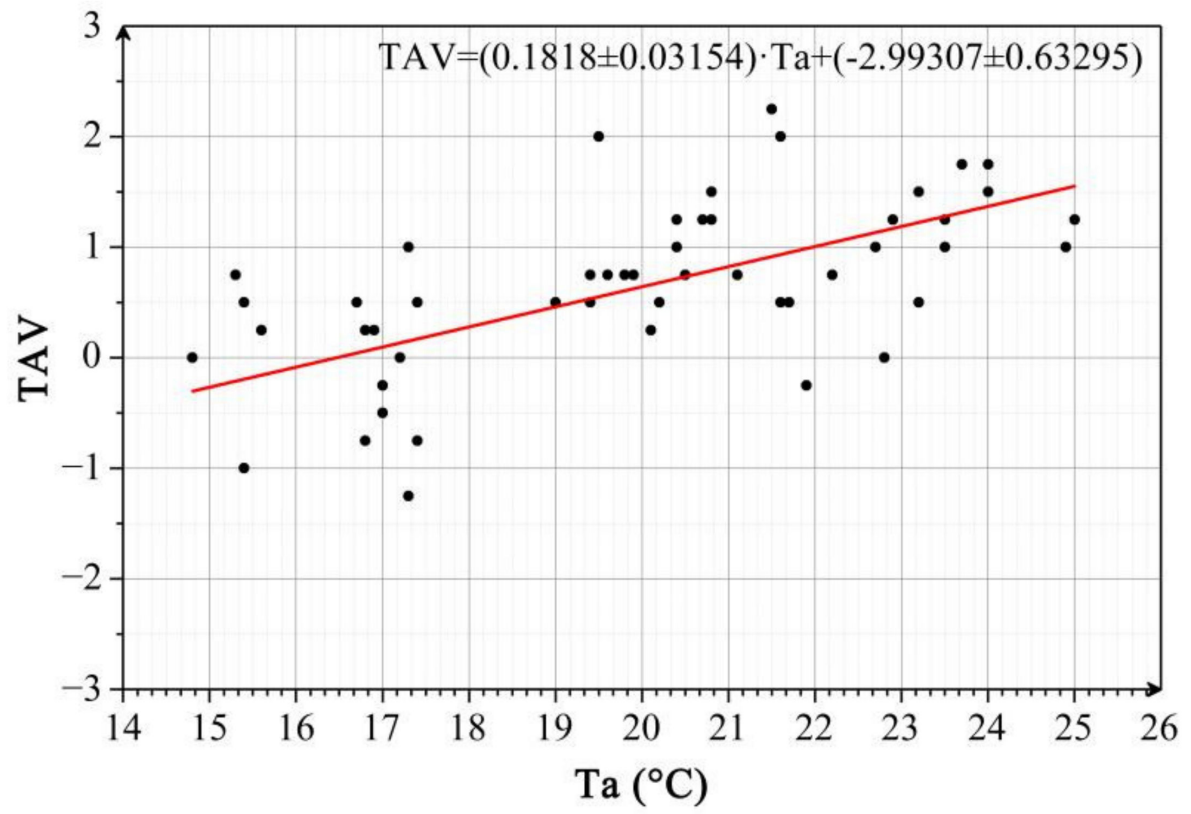

Figure 13. The quantitative relationship between TAV and Ta.

\subsubsection{Comparison of Actual Thermal Sensation and Predicted Thermal Sensation}

In this study, the PMV model proposed by P.O. Fanger was also applied to compare the similarities and differences between the actual thermal sensation and the predicted thermal sensation, of which would be analyzed through the comparison followed.

Figure 14 illustrates the relationship between TSV, PMV and air temperature (Ta). It can be found that there was a strong linear relationship between TSV, PMV and Ta, while the difference between them was small. However, according to related studies, the subject 
students were informed of the actual temperature during the actual heat sensory voting, while the PMV model was based on the condition that the tested students did not know the actual environmental parameters. Therefore, it was possible that the symmetry of information about the environmental parameters weakened the impact of psychological effects on heat sensory voting [49].

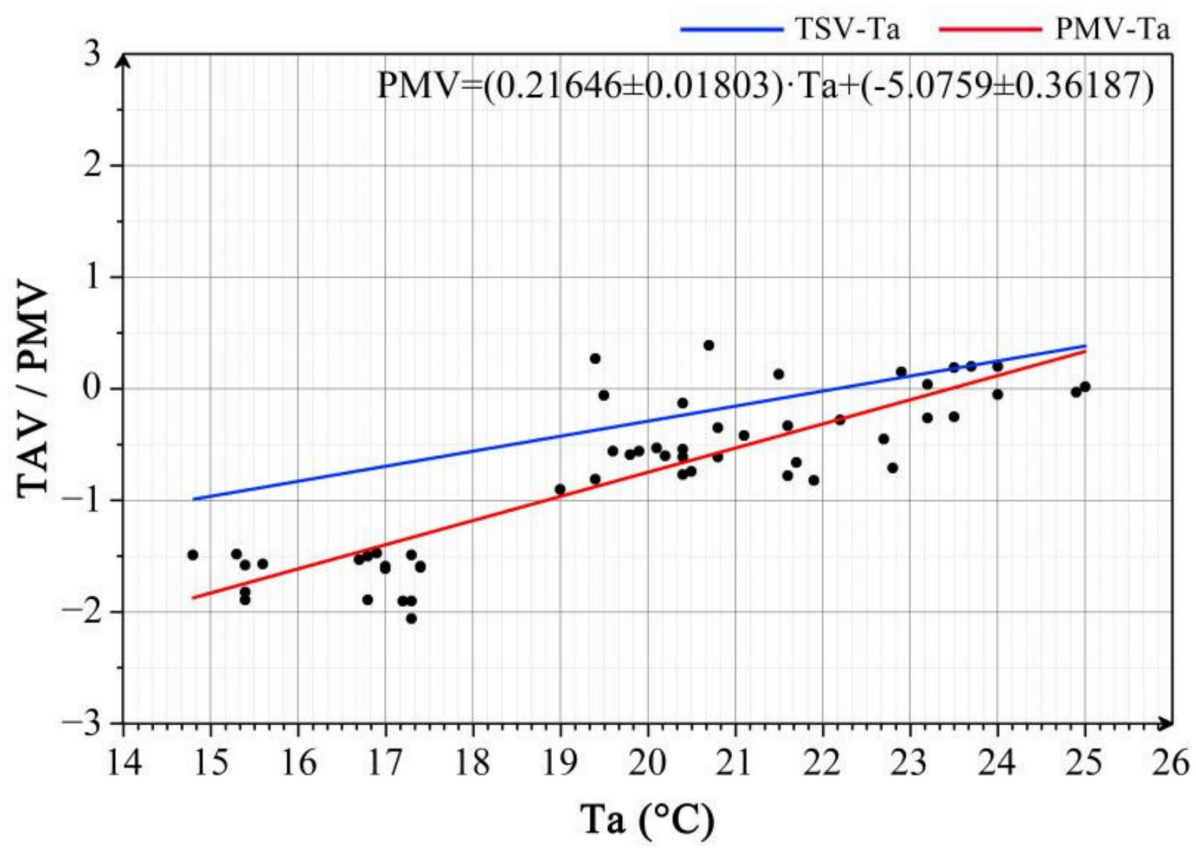

Figure 14. The quantitative relationship between PMV and Ta.

\subsection{Data Analysis of Learning Efficiency Test}

\subsubsection{Processing and Data Analysis}

SPSS 20.0 and Analysis of Variance (ANOVA) software were used in this study to process and analyze the data obtained from the learning efficiency tests. Then, the existence of significant differences between similar data under each experimental environmental condition could be judged while setting the significance level at $0.05(p<0.05)$. However, simply calculating the $p$ was not enough to show the magnitude of the difference in results. Therefore, in this study, the Effect Size (ES) was also calculated to analyze the strength of the experimental effects [50].

In ANOVA, the ES value can be calculated from Equation (3).

$$
E S=\sqrt{\frac{F}{\mathrm{n}}}
$$

where the test statistic $\mathrm{F}$ is ANOVA, $\mathrm{n}$ is the number of people in each group if the groups are equal, and if the groups are not equal, the summed mean needs to be calculated. Moreover, the quantification of ES has been developed by related studies, which represent three tiers of weaker, moderate and stronger impact at ES of $0.1,0.25$ and 0.4 , respectively [51].

The final results of the ACP, ART and LP as well as P and ES of the relevant test items were calculated as shown in Tables A3-A6 in Appendix A. The ANOVA showed that the $p$-values were greater than 0.05 for all tests except Stereo Vision and Visual Learning, indicating that for most tests, there were no significant differences in the experimental results between the weather and classroom type conditions. According to other studies, similar error cases have been observed, which means that there were always $1-2$ test items with $p$-values less than 0.05 in the calculation of $p$-values for multiple test items [52-54].

In addition, for all test items, their ES values were greater than 0.1 , and most of the test items achieved ES values of about 0.25 , showing that different weather and classroom 
types of conditions affect all test items, but to a different extent for each test item. Among them, the different weather and classroom types had a moderate effect on the ART for most of the test items, except for the weak effect on the ART for Schulte Grid, Number Filter and Logical Events. In terms of the degree of impact on ACP, the impact on Memory Scanning and Continuous Addition \& Subtraction was weak, but with moderate effects on the other test items.

In conclusion, under different weather conditions and classroom-types, there were differences in the degree of influence on different test items in this study. There were also differences in the degree of influence of ART and ACP on the same test items.

3.3.2. Relationship between Learning Efficiency and Temperature in the Four Dimensions

In this study, the learning efficiency evaluation method applied, namely the APCD, contained four dimensions: Perceptual Ability, Concentration Ability, Memory Ability and Thinking Ability. The relevant test contents and methods were given in detail above, and the results were standardized to obtain the changes in the four dimensions of the tested students' learning during the day, as shown in Figures 15-17. The points in the figures were all processed averages for each test item, with ACP, ART and LP indicating average correctness, average reaction time and learning performance, respectively.
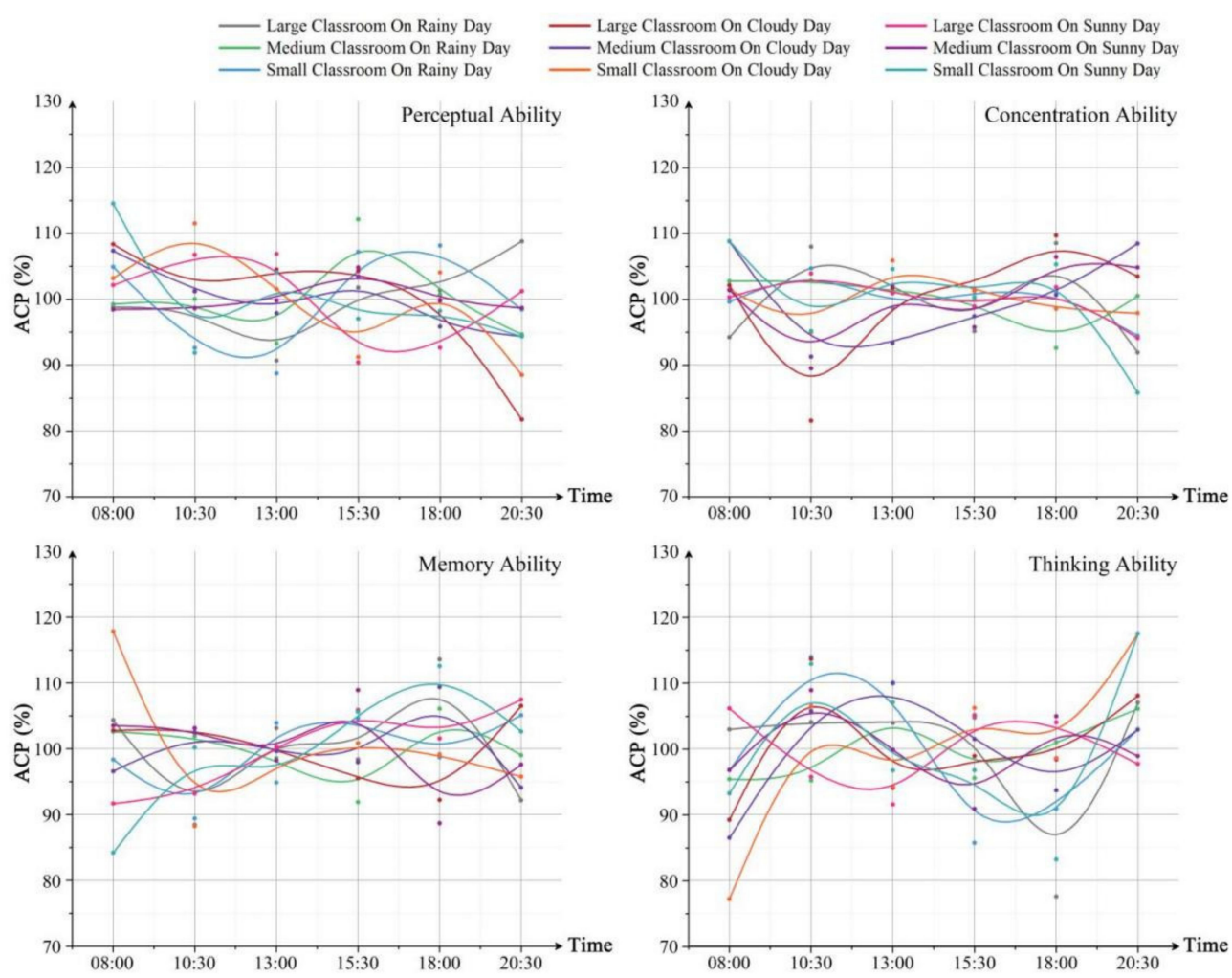

Figure 15. The ACP of the four dimensions in the three classroom-types under different weather conditions throughout the day. 

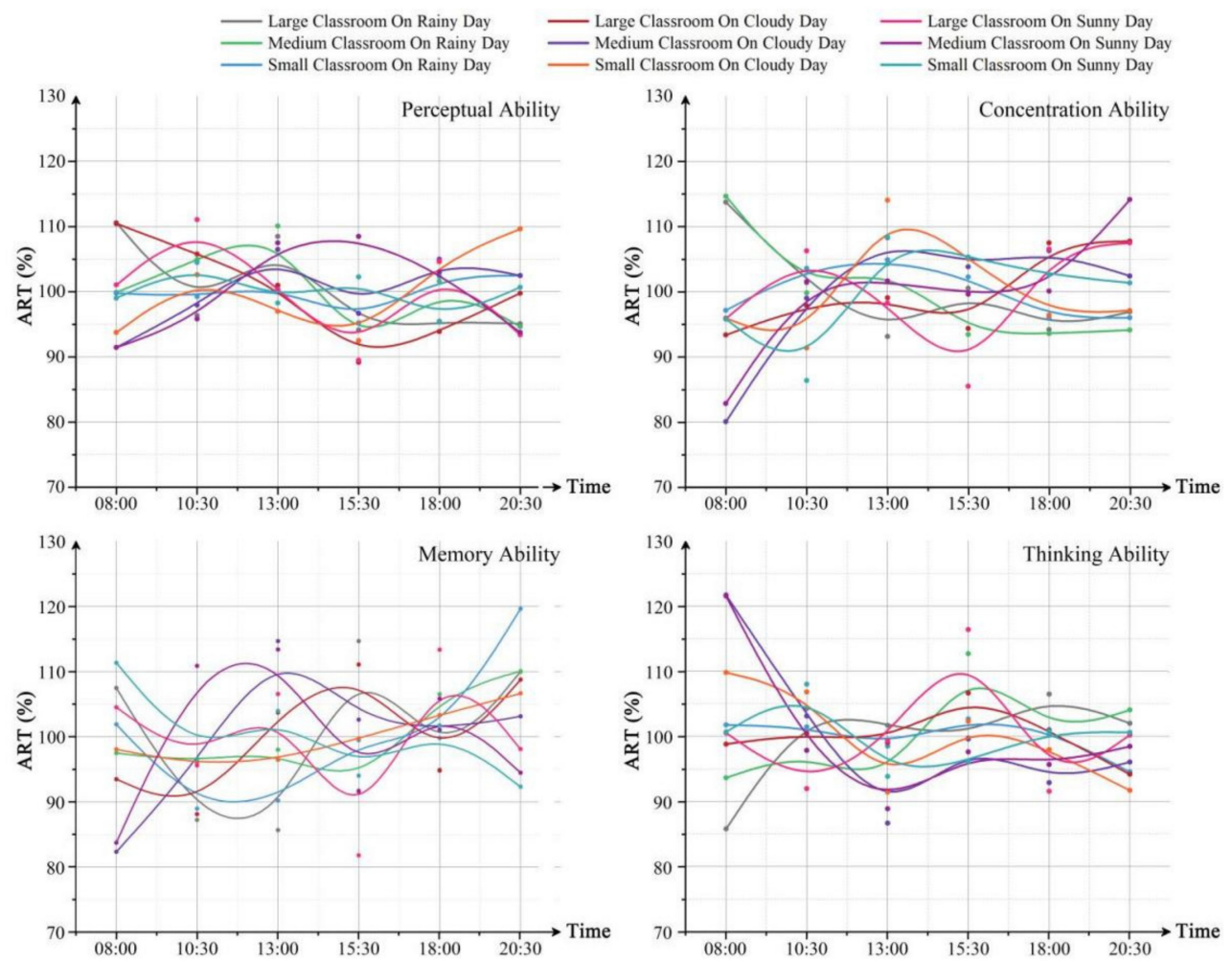

Figure 16. The ART of the four dimensions in the three types of classrooms under different weather conditions throughout the day.

As shown in Figure 15, in terms of correctness, the ACP showed a more pronounced trend of repeated fluctuations throughout the day, regardless of the different weather conditions or the different classroom-types. However, the items of Thinking Ability test tended to increase, then decrease, and then increase again, while the rest of the test items showed the opposite trend. Simultaneously, as indicated in Figure 16, the ART also showed more pronounced repeated fluctuations throughout the day in terms of response time. However, in both Memory Ability and Thinking Ability tests, the ART tended to decrease, then increase, then decrease. In the case of Perceptual Ability and Concentration Ability tests, the RT showed the opposite trend. Generally, the RT of the Perceptual Ability test and Thinking Ability test varied more, while the RT of the remaining two tests varied less. In other words, Figure 17 presents the LP of the four types of tests, and it can be found that their trend of changes was exactly opposite to the ART. In addition, the LP of the Perceptual Ability and Thinking Ability tests varied relatively more, while the concentration Ability and Memory Ability tests varied relatively less.

As is evident, when exploring student learning effectiveness, it is inappropriate to look at only one type of ability performance. This is because various types of test items are affected to different degrees under different conditions, and it is one-sided and error-prone to judge students' learning efficiency using only the test results of a single category. 

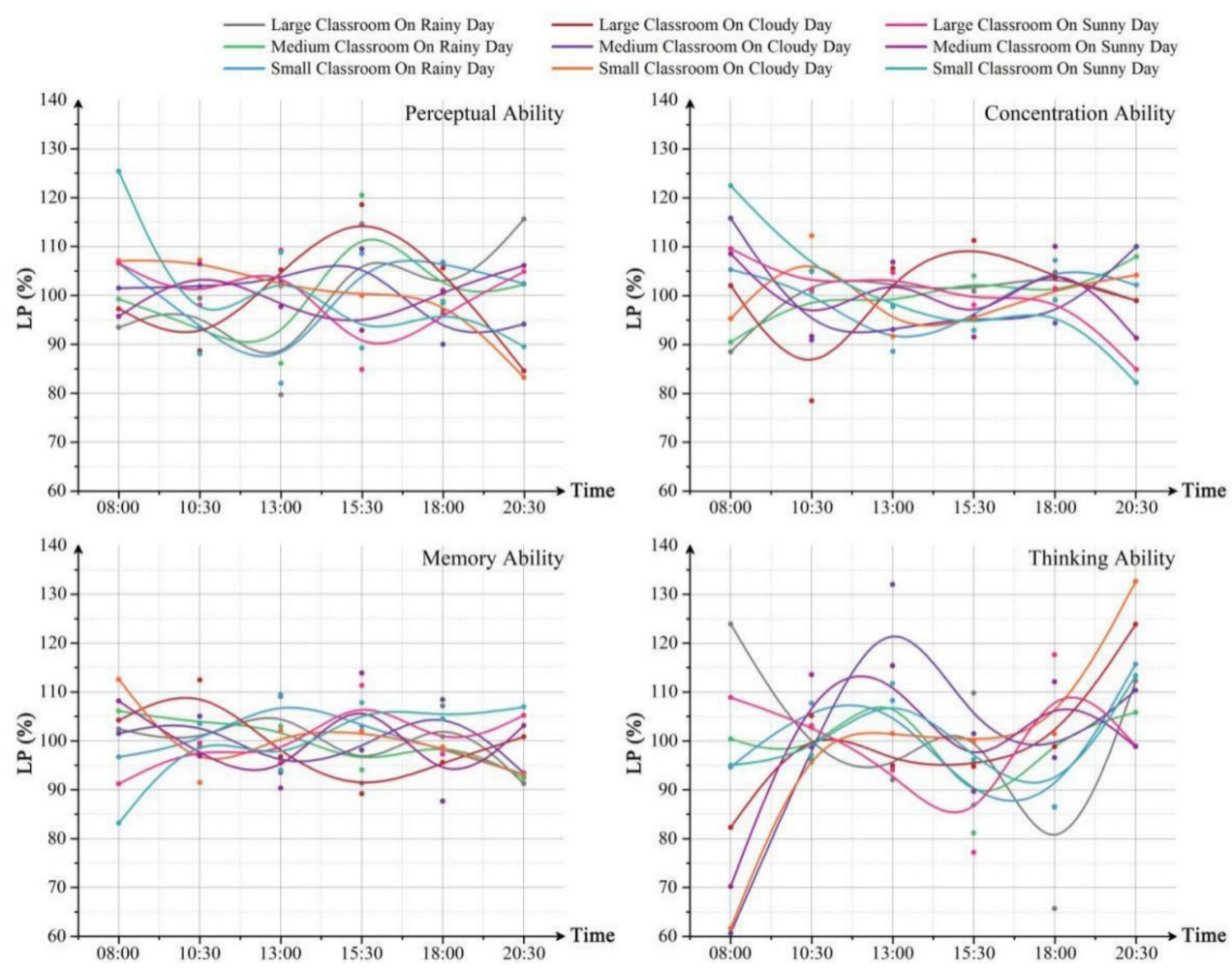

Figure 17. The LP of the four dimensions in the three classroom-types under different weather conditions throughout the day.

\section{Results and Discussion}

\subsection{Relationship between Integrated Learning Efficiency and Temperature}

The results of the combined learning efficiency test for the tested students under three weather conditions and three classroom-types were shown in Figures 18-20. In each figure, nine curves indicate the trends of learning efficiency indicators under different conditions. Each point on the curve indicates the average test data of the tested students.

As indicated in Figure 18, the ACP of the combined learning efficiency of the tested students increased firstly and then decreased throughout the day, reaching the highest value at around 13:30, which was the highest temperature moment of the day. Furthermore, the magnitude of change in the ACP was greatest on sunny days, followed by rainy days, and the smallest on cloudy days. This is due to the greater variation in indoor temperature under sunny conditions and the least variation in indoor temperature under cloudy conditions. In the same weather conditions, the variation in ACP was greater in the small classroom than in the others, also because the indoor temperature varied more in the small classroom.

Figure 19 illustrates the change in ART for the combined learning efficiency of the tested students across conditions, with a rapid decrease followed by a small increase. In particular, the ART fluctuated around 95\% during the time period of 10:30 to 18:00, which was the shortest interval throughout the whole day.

As for the combined learning performance, as presented in Figure 20, the LP under different conditions showed a consistent trend of increasing and then decreasing during the whole day. The LP-value was highest at around 13:30. Additionally, under different weather conditions, the LP varied the most under sunny conditions, followed by cloudy conditions, and the least under rainy conditions. In contrast, under the same weather conditions, the LP varied the most in the small classroom, followed by the medium classroom, and the least in the large classroom. The reasons for the above were consistent with the reasons for the variation in the ART. Simultaneously, combining all the learning performance data, it 
was found that nearly half of the $\mathrm{L} p$-values were greater than $100 \%$, which indicated that the tested students in this condition performed above their average learning performance.

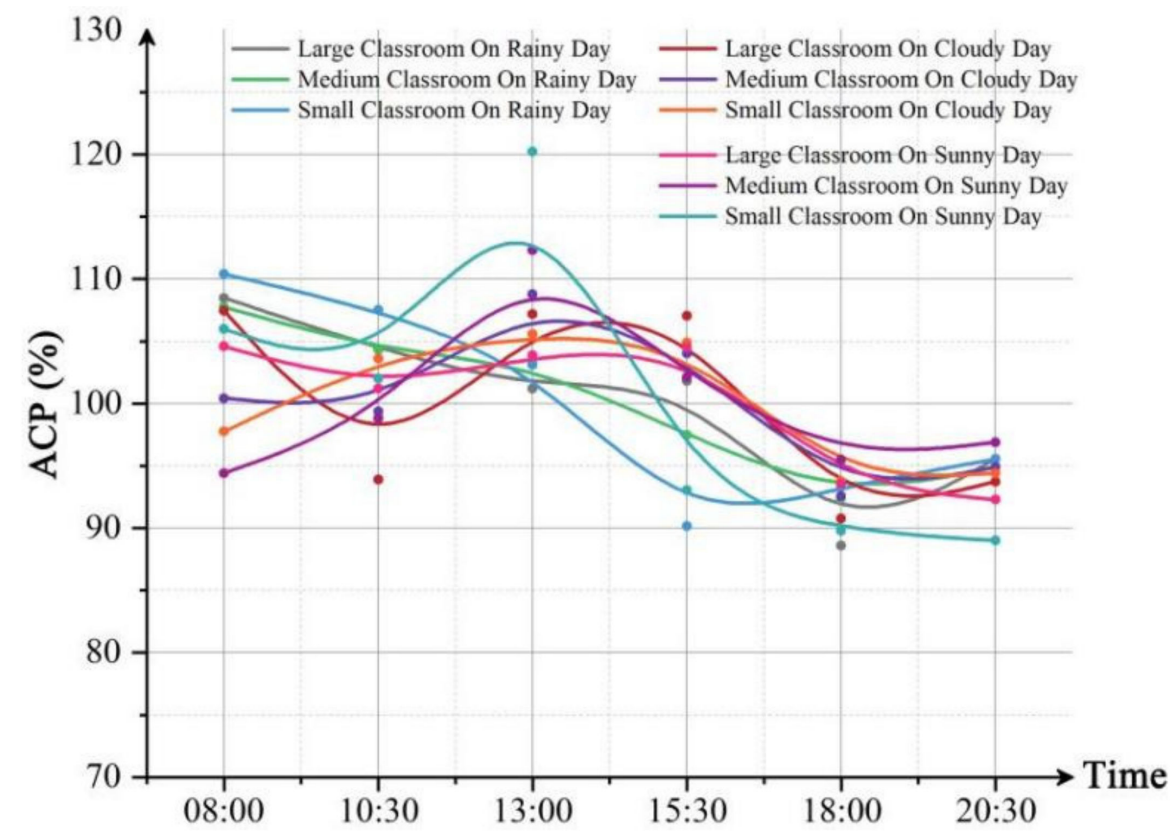

Figure 18. The ACP of the learning efficiency test in the three classroom-types under different weather conditions throughout the day.

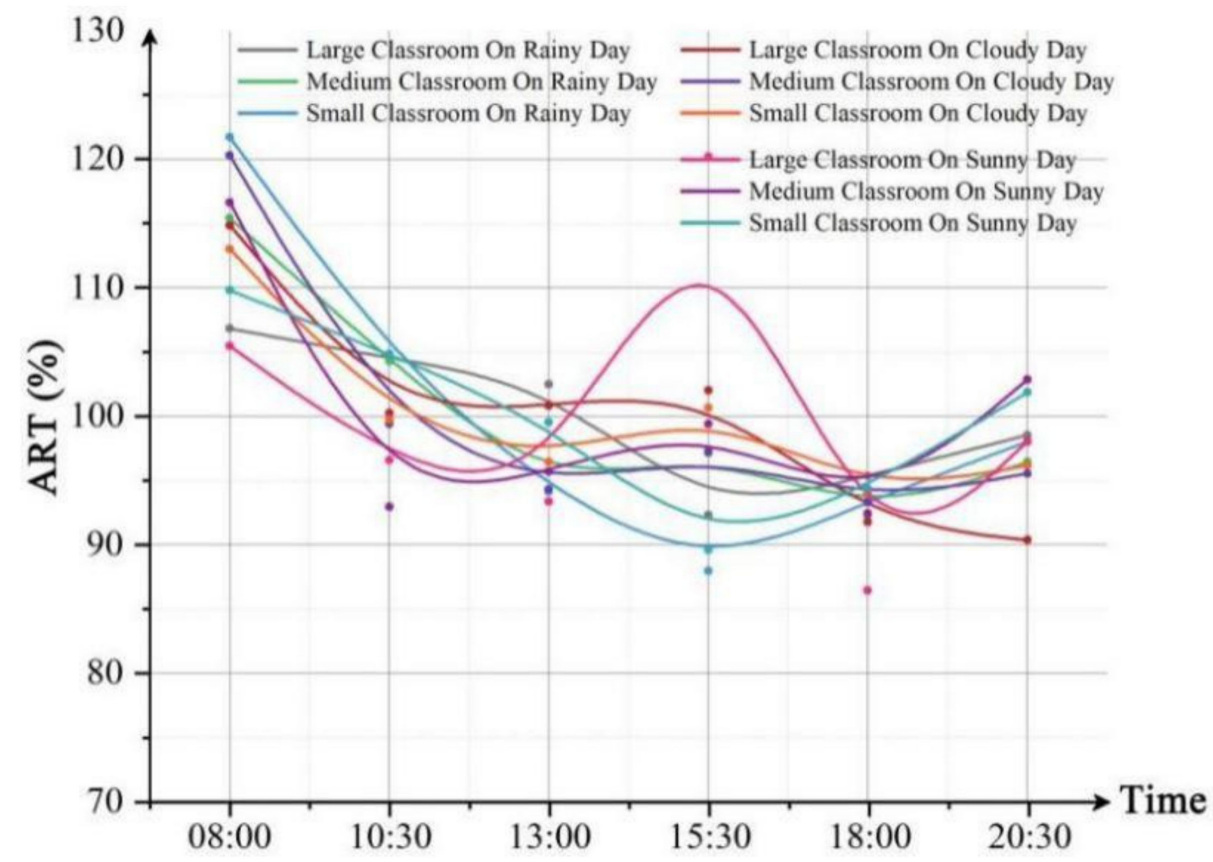

Figure 19. The ART of the learning efficiency test in the classroom-types under different weather conditions throughout the day. 


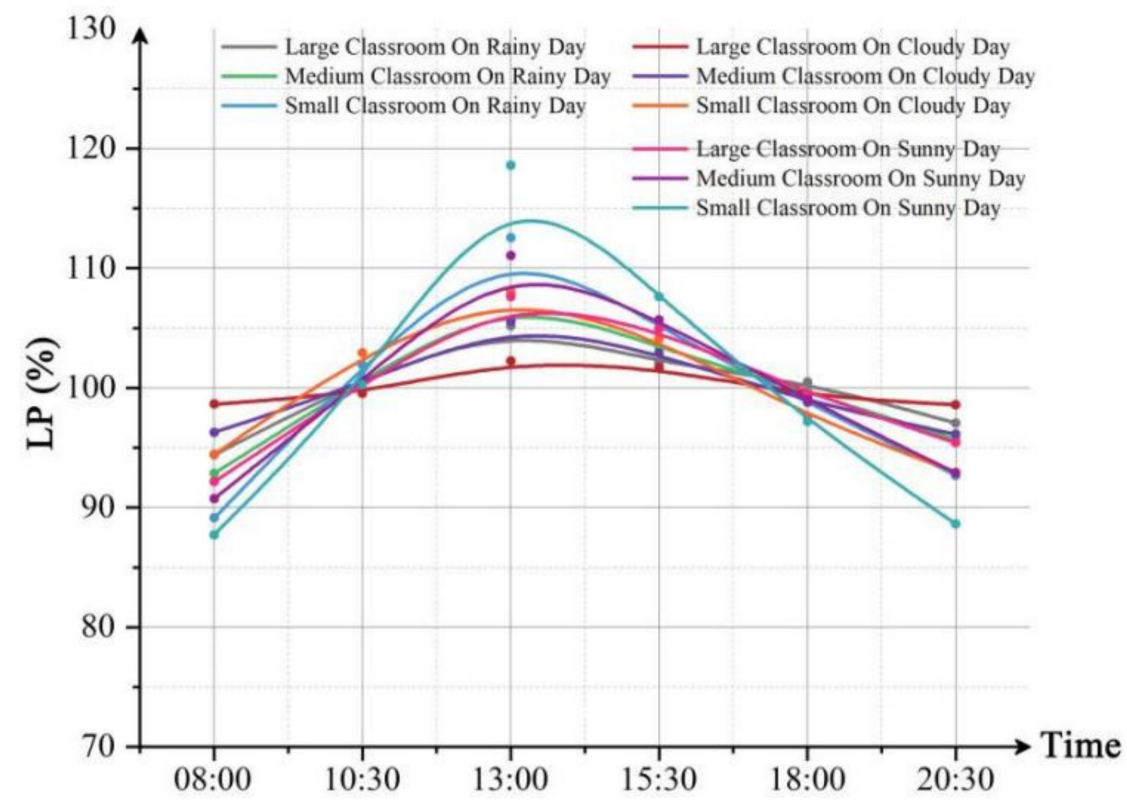

Figure 20. The LP of the learning efficiency test in the three classroom-types under different weather conditions throughout the day.

Conclusively, it was found that under different conditions, when the time was around 13:00, the tested students had the highest ACP, the shortest ART and the highest LP. It indicated that during the whole day in the transition season, the learning efficiency was relatively the highest at that time. Influenced by different outdoor temperatures, the students' learning efficiency varied the most under different weather conditions on sunny days, followed by rainy days and the least on cloudy days. Additionally, influenced by different indoor temperatures, the students' learning efficiency varied most in small classrooms, the second most in medium classrooms and the least in large classrooms under the same weather conditions.

\subsection{Relationship between Thermal Comfort and Learning Performance}

As can be seen through the circumstances investigated in this study, accurately judging the comfort level of an environment is relatively easy for people to do, but judging their own learning efficiency in that environment is more difficult. Subjective evaluations of learning efficiency often present a large margin of error and are unconvincing due to personal factors. Therefore, if the quantitative relationship between people's evaluations of thermal comfort and learning efficiency can be derived from experimental data, the human thermal comfort can be improved to some extent by changing the conditions of the surrounding environment, while improving learning efficiency. Nevertheless, the analysis of the relationship between the ACP, RT and thermal comfort alone is one-sided, and only the choice of the LP, a comprehensive learning efficiency index, can more accurately represent the learning efficiency of the tested students. Thus, in the study of the relationship between students' thermal comfort and learning efficiency in different classrooms during the transition season, the LP was selected to be explored.

Firstly, the relationship between TSV and LP is shown in Figure 21. This linear function combined all the results of the test items in the four dimensions, obtained by fitting the mean of each students' TSV to its corresponding LP data in a regression analysis. It can be seen that the LP shows a slow increase when the TSV changes from Cold (-3) to Hot (+3). In fact, according to the ASHRAE Standard 55 [4], the TSV is "neutral" at a temperature of approximately $26.5^{\circ} \mathrm{C}$. Therefore, the LP tended to improve with increasing temperature in the transition season because the indoor temperature of classrooms ranged from approximately $14{ }^{\circ} \mathrm{C}$ to $25^{\circ} \mathrm{C}$, which were lower than $26.5^{\circ} \mathrm{C}$. Overall, it can be interpreted that 
for indoor environments in classrooms during the transition season, students were more productive in warmer environments.

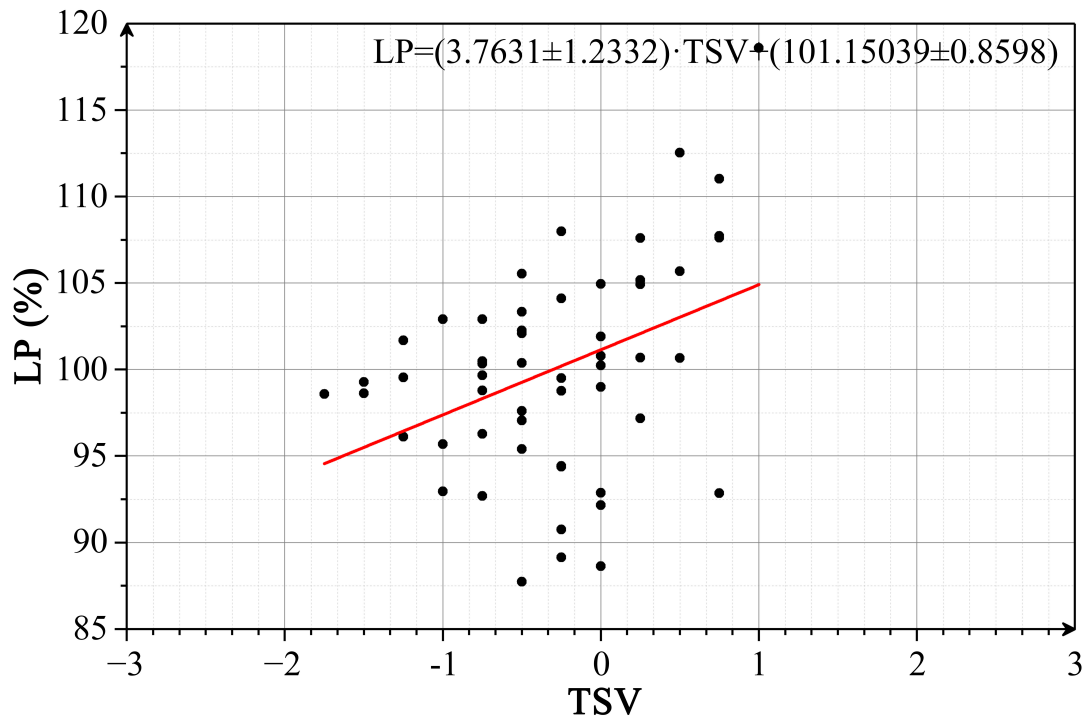

Figure 21. The quantitative relationship between TSV and LP.

Secondly, the relationship between PMV and LP is presented in Figure 22. The results were similar to those of the TSV-LP, but there was some error. This is probably because the subject students were informed of the actual temperature before the actual heat sensation voting, and the PMV model was built under the condition that the tested students didn't know the actual environmental parameters. Therefore, the error could be a psychological effect of the asymmetry of information on the students during the actual heat sensation voting.

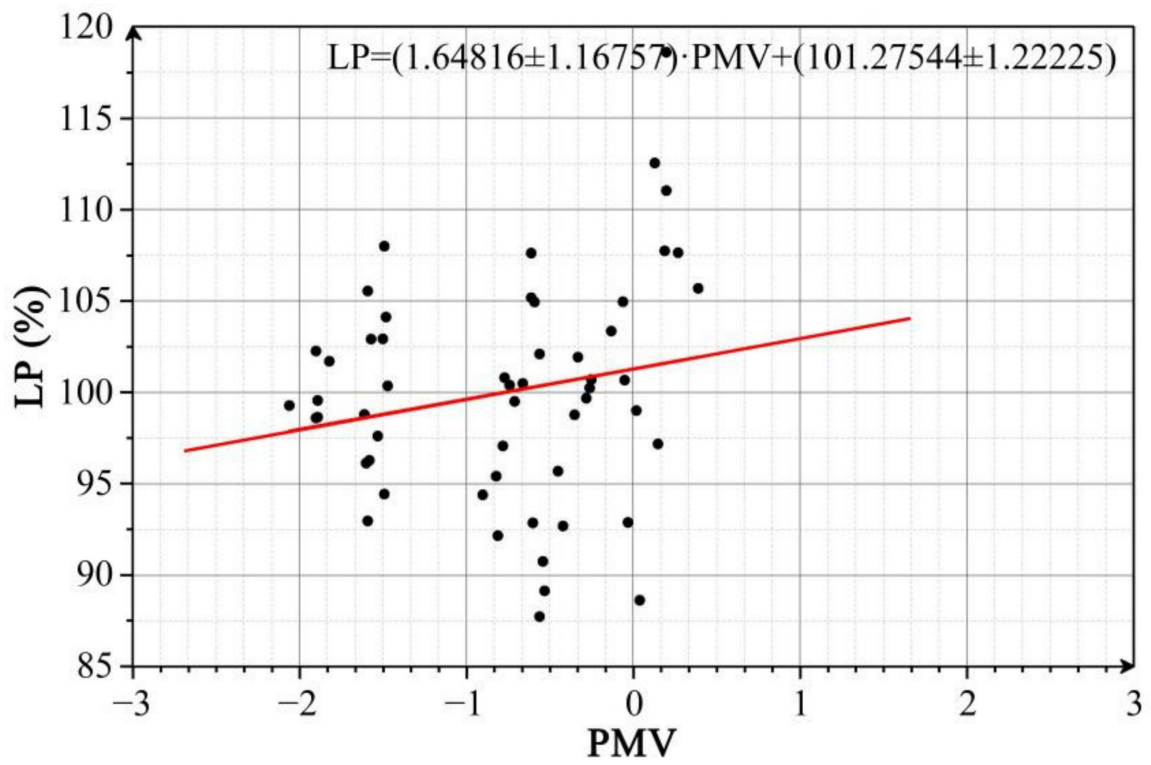

Figure 22. The quantitative relationship between PMV and LP.

Thirdly, the relationship between TAV and LP is indicated in Figure 23. It can be clearly seen that the TAV of the tested students showed a linear relationship with LP. When the TAV changed from "Very Dissatisfied" (-3) to "Very Satisfied" (+3), the LP showed an increasing trend, indicating that the higher the TAV, the better the LP. 


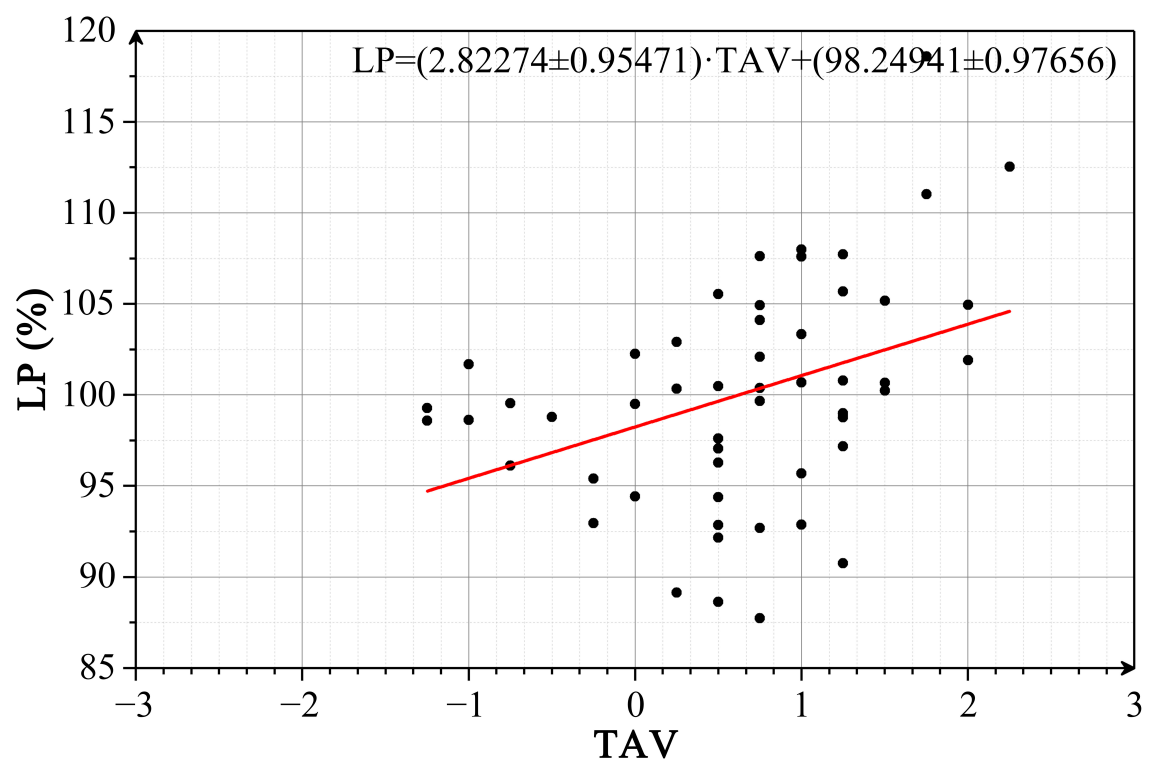

Figure 23. The quantitative relationship between TAV and LP.

\section{Conclusions}

In this study, the current research progress and results of domestic and international scholars on human thermal comfort and learning efficiency of students in the classroom were reviewed and summarized. Most of the research experiments were found to be simulations that were conducted under artificially set environmental conditions, with little relation to natural conditions. Additionally, due to the small sample sizes and single measurement variables, there always were many errors in the experimental results. In order to remedy these shortcomings, this study established the relationship between thermal comfort and learning efficiency of the tested students through the thermal comfort questionnaire and learning efficiency test under typical natural conditions in the transition season. Furthermore, the correlation between the current state of indoor environmental conditions, thermal comfort and learning efficiency was analyzed to provide guidance for improving indoor thermal conditions. The following three main conclusions were obtained.

- Under the natural conditions of the transitional season, the indoor temperature of the classrooms varied between $14{ }^{\circ} \mathrm{C}$ and $25^{\circ} \mathrm{C}$ throughout the day and was higher between 12:00 and 13:30. Meanwhile, the TSV of the subject students varied between -3 and +3 , and the highest TAV was obtained around 13:00. On the one hand, under different weather conditions, sunny days with higher temperatures saw relatively higher TAV, cloudy days were second and rainy days saw the lowest. On the other hand, under the same weather conditions, small classrooms with higher indoor temperatures had the highest TAV, medium classrooms the second highest, and large classrooms the lowest. These can indicate that during the transition season, the subject students preferred a warmer environment.

- Under the natural conditions of the transitional season, different weather and classroom type conditions have different degrees of influence on each test item of learning effectiveness. Then, under the natural condition that the indoor temperature of the classroom was between $14{ }^{\circ} \mathrm{C}$ and $25^{\circ} \mathrm{C}$ in the transition season, student $\mathrm{LP}$ exhibited a trend of increasing and then decreasing throughout the day, with the highest LP at around 13:30. Furthermore, under different weather conditions, student's LP varied the most under sunny conditions with higher indoor temperatures, followed by cloudy days and the least under rainy days. However, under the same weather conditions, the small classrooms with higher indoor temperatures showed the greatest variation 
in the LP, followed by the medium classrooms, and the large classrooms showed the least variation.

- Under natural conditions of the transition season, and LP was linearly related to the TSV and TAV. On the one hand, the LP showed a slow upward trend as the subject student's TSV changed from cold $(-3)$ to warm $(+3)$. On the other hand, the LP presented an increasing trend when the TAV of the subject students changed from very dissatisfied $(-3)$ to very satisfied $(+3)$.

Combined with the analysis of the correlation between the current state of different classroom environmental conditions and thermal comfort and learning efficiency, it was found that changes in classroom indoor environmental conditions impact student learning efficiency. Simultaneously, the causes of the adverse effects are not only common problems such as poor insulation performance of the envelope, but also factors such as a large shape coefficient and large window-to-floor ratio. In response to the above problems, this paper proposes the following improvement strategies.

- For the walls, which account for the largest proportion of the building envelope, there is a need to improve their insulation performance. Firstly, in the building construction process, a single insulation material such as aerated concrete or a multifunctional conforming wall composed of high-efficiency insulation materials should be vigorously used, which has the advantages of high strength and heat insulation compared with ordinary brick walls. Secondly, to avoid the formation of internal insulation and sandwich insulation trims panel cracking, "thermal bridge", moisture and other problems, it is recommended to use the construction form of external insulation to reduce indoor heat loss and improve the thermal insulation performance of the wall.

- For windows, the weakest part of the envelope, it is equally important to improve their insulation performance. Firstly, the window-to-wall ratio of the classroom needs to be controlled. The window-to-wall ratios of the north, east-west and south directions are controlled to reduce the heat loss in the room. Secondly, the airtightness of the windows needs to be improved to reduce heat loss due to air infiltration. Window profiles with better cross-sectional dimensions and rigidity can be selected, and window installation can be performed with a softer mass and a greater compression ratio seal. Thirdly, using double-pane windows, multi-pane windows or low-E glass windows and other new heat transfer coefficients are also effective ways to insulate windows.

- For classrooms with roof panels, it is equally important to improve the insulation performance of the roof. Using lightweight, non-absorbent or less absorbent insulation materials as roofing materials, with the "inverted" roofing as the main construction practices, can effectively improve the insulation and waterproof performance of the roof.

- For the classrooms with small window spaces, their indoor lighting and solar radiation gains are often less. Thus, it is also important to improve the solar radiation heat gain of this type of classroom, which can be achieved by means of an external sunroom and thermal storage walls.

It is important to mention that this paper explores the relationship between changes in students' thermal comfort caused by changes in indoor environmental conditions and students' own learning efficiency under typical natural conditions during the transition season. However, according to the relevant standards, the temperature of about $26.5^{\circ} \mathrm{C}$ when the thermal sensation is "neutral", where the temperature is a critical value theoretically. In this paper, the actual classroom experimented, however, had an indoor temperature range of $14^{\circ} \mathrm{C}$ to $25^{\circ} \mathrm{C}$, and we could not find out how the learning efficiency changed at higher temperatures. In addition, adopting a holistic approach to explore all aspects of thermal comfort in Indoor Environmental Quality, including visual and auditory comfort, which will be more comprehensive and objective. More thought and experimental analysis are needed for research and practice in these areas. 
Author Contributions: Conceptualization, X.M.; Data curation, Z.Z., X.C. and Y.C.; Formal analysis, X.M.; Funding acquisition, H.L.; Investigation, X.M., Z.Z. and X.C.; Methodology, H.L.; Project administration, Y.C.; Software, Z.Z. and X.C.; Validation, Y.C.; Visualization, Z.Z.; Writing-original draft, X.M.; Writing-review \& editing, H.L. and S.K. All authors have read and agreed to the published version of the manuscript.

Funding: This research received no external funding.

Institutional Review Board Statement: Not applicable.

Informed Consent Statement: Informed consent was obtained from all subjects involved in the study.

Data Availability Statement: Data was contained within supplementary material.

Conflicts of Interest: The authors declare no conflict of interest.

\section{Appendix A}

Table A1. Basic information of the tested students.

\begin{tabular}{|c|c|c|c|c|}
\hline \multirow{2}{*}{ Test Week } & \multirow{2}{*}{ Classroom Type } & \multicolumn{2}{|c|}{ Gender } & \multirow{2}{*}{ Mean Age } \\
\hline & & Male & Female & \\
\hline \multirow{4}{*}{$\begin{array}{l}\text { Week1 (1 March 2021-7 March 2021) } \\
\text { Week2 (8 March 2021-14 March 2021) }\end{array}$} & Small Classroom & 10 & 10 & 22.75 \\
\hline & Medium Classroom & 10 & 10 & 22.50 \\
\hline & Large Classroom & 10 & 10 & 22.75 \\
\hline & Small Classroom & 10 & 10 & 23.00 \\
\hline Week3 (15 March 2021-21 March 2021) & Medium Classroom & 10 & 10 & 22.75 \\
\hline \multirow[t]{2}{*}{ Week4 (22 March 2021-28 March 2021) } & Large Classroom & 10 & 10 & 22.75 \\
\hline & Small Classroom & 10 & 10 & 22.20 \\
\hline \multirow{3}{*}{$\begin{array}{l}\text { Week5 (29 March 2021-4 April 2021) } \\
\text { Week6 (5 April 2021-11 April 2021) }\end{array}$} & Medium Classroom & 10 & 10 & 22.00 \\
\hline & Large Classroom & 10 & 10 & 22.75 \\
\hline & Small Classroom & 10 & 10 & 22.40 \\
\hline \multirow{2}{*}{$\begin{array}{l}\text { Week7 (12 April 2021-18 April 2021) } \\
\text { Week8 (19 April 2021-25 April 2021) }\end{array}$} & Medium Classroom & 10 & 10 & 22.00 \\
\hline & Large Classroom & 10 & 10 & 23.00 \\
\hline \multirow{4}{*}{$\begin{array}{l}\text { Week9 (26 April 2021-2 May 2021) } \\
\text { Week10 (3 May 2021-9 May 2021) }\end{array}$} & Small Classroom & 10 & 10 & 22.25 \\
\hline & Medium Classroom & 10 & 10 & 22.75 \\
\hline & Large Classroom & 10 & 10 & 23.00 \\
\hline & Small Classroom & 10 & 10 & 22.75 \\
\hline \multirow{3}{*}{$\begin{array}{l}\text { Week11 (10 May 2021-16 May 2021) } \\
\text { Week12 (17 May 2021-23 May 2021) }\end{array}$} & Medium Classroom & 10 & 10 & 22.50 \\
\hline & Large Classroom & 10 & 10 & 22.50 \\
\hline & Small Classroom & 10 & 10 & 22.20 \\
\hline \multirow{2}{*}{ Week13 (24 May 2021-30 May 2021) } & Medium Classroom & 10 & 10 & 22.00 \\
\hline & Large Classroom & 10 & 10 & 22.75 \\
\hline
\end{tabular}

Table A2. The corresponding methods of operation for main test items.

\begin{tabular}{|c|c|c|}
\hline Test Item & Purposes & Operation Method \\
\hline Letter Search & Basic Visual Perception & $\begin{array}{l}\text { Faced with a card printed with ten capital letters without repetition and a } \\
\text { target letter, the test students judge whether the target letter appears in the } \\
\text { ten letters [40]. }\end{array}$ \\
\hline $\begin{array}{l}\text { Word Color } \\
\text { Interference }\end{array}$ & $\begin{array}{l}\text { Color Perception and } \\
\text { Semantic Recognition } \\
\text { Ability }\end{array}$ & $\begin{array}{l}\text { Get a piece of text printed with multiple description colors, each word is } \\
\text { printed in a different color, and the students choose the item with the same } \\
\text { semantics and colors [41]. }\end{array}$ \\
\hline Graphic Overlay & $\begin{array}{l}\text { Spatial Reasoning and } \\
\text { Perception }\end{array}$ & $\begin{array}{l}\text { Seven plane figures are randomly superimposed, and the test students } \\
\text { judge the figure names in order from top to bottom according to the edge } \\
\text { shape of the superimposed graphics [42]. }\end{array}$ \\
\hline Stereo Vision & Spatial Perception & $\begin{array}{l}\text { A variety of three-dimensional figures are rotated at any angle, and the } \\
\text { students judged from the front view that the cube has several faces [43]. }\end{array}$ \\
\hline
\end{tabular}


Table A2. Cont.

\begin{tabular}{|c|c|c|}
\hline Test Item & Purposes & Operation Method \\
\hline Schulte Grid & Concentration & $\begin{array}{l}\text { Randomly fill } 25 \text { Arabic numbers in a } 5 \times 5 \text { grid, and the test students need } \\
\text { to find the number } 25 \text { in sequence starting from the number } 1 \text { [ } 44] \text {. }\end{array}$ \\
\hline Digital Filter & $\begin{array}{l}\text { Pay Attention to the } \\
\text { Ability to Select Features }\end{array}$ & $\begin{array}{l}\text { Randomly select any number from } 10 \text { Arabic numerals to form a set of } \\
\text { 32-digit numbers. From the number column, the test students find all } \\
\text { adjacent numbers whose sum is } 10 \text {. }\end{array}$ \\
\hline Visual Learning & $\begin{array}{l}\text { Memory Recognition } \\
\text { Ability }\end{array}$ & $\begin{array}{l}\text { Four meaningless figures appear anywhere in the } 3 \times 3 \text { grid, each figure } \\
\text { appears two or three times, and the nine spaces are filled. The test students } \\
\text { need to memorize the position of each figure in the grid within } 20 \mathrm{~s} \text { and } \\
\text { reproduce it in a blank table [40]. }\end{array}$ \\
\hline Memory Scanning & Memory Neurobehavior & $\begin{array}{l}\text { The test students first have } 5 \mathrm{~s} \text { to look at a set of numbers carefully, and } \\
\text { then give another set of numbers, and the students are asked to find the } \\
\text { repeated number in the two sets of numbers [45]. }\end{array}$ \\
\hline Logical Events & Logical Reasoning Ability & $\begin{array}{l}\text { Each test item gives five short description events, and asks students to } \\
\text { choose the most logical one from the options [46]. }\end{array}$ \\
\hline $\begin{array}{l}\text { Continuous Addition } \\
\quad \& \text { Subtraction }\end{array}$ & Assignment & $\begin{array}{c}\text { The ten numbers are arranged in a random order, and the addition and } \\
\text { subtraction symbols are inserted between two adjacent numbers, and the } \\
\text { students are required to calculate the result. }\end{array}$ \\
\hline
\end{tabular}

Table A3. The final results of the ACP, ART and LP of the relevant test items on rainy days.

\begin{tabular}{|c|c|c|c|c|}
\hline \multirow{3}{*}{ Test Item } & \multirow{3}{*}{ Indicator } & \multicolumn{3}{|c|}{ Test Conditions } \\
\hline & & \multicolumn{3}{|c|}{ Sunny Days } \\
\hline & & Small Classroom & Medium Classroom & Large Classroom \\
\hline \multirow{4}{*}{ Letter Search } & $\mathrm{ACP}$ & $77.71 \pm 9.20$ & $81.95 \pm 9.48$ & $80.63 \pm 9.30$ \\
\hline & ART & $25.88 \pm 1.41$ & $23.79 \pm 1.12$ & $22.63 \pm 0.74$ \\
\hline & LP & 3.00 & 3.44 & 3.56 \\
\hline & $\mathrm{ACP}$ & $78.61 \pm 10.21$ & $92.15 \pm 6.48$ & $79.10 \pm 9.01$ \\
\hline \multirow[t]{2}{*}{ Word Color Interference } & ART & $27.54 \pm 5.17$ & $20.42 \pm 1.81$ & $22.71 \pm 2.65$ \\
\hline & LP & 2.85 & 4.51 & 3.48 \\
\hline \multirow{4}{*}{ Graphic Overlay } & $\mathrm{ACP}$ & $84.84 \pm 9.96$ & $77.76 \pm 13.03$ & $85.10 \pm 11.18$ \\
\hline & ART & $34.29 \pm 5.09$ & $33.13 \pm 3.73$ & $31.29 \pm 5.67$ \\
\hline & LP & 2.47 & 2.35 & 2.72 \\
\hline & $\mathrm{ACP}$ & $83.54 \pm 10.27$ & $93.13 \pm 8.04$ & $83.68 \pm 10.31$ \\
\hline \multirow[t]{3}{*}{ Stereo Vision } & ART & $13.88 \pm 2.08$ & $10.21 \pm 0.85$ & $11.21 \pm 1.44$ \\
\hline & LP & 6.02 & 9.12 & 7.47 \\
\hline & $\mathrm{ACP}$ & $90.82 \pm 8.38$ & $92.85 \pm 6.09$ & $84.86 \pm 11.08$ \\
\hline \multirow[t]{2}{*}{ Schulte Grid } & ART & $49.21 \pm 4.63$ & $35.83 \pm 4.19$ & $38.57 \pm 3.33$ \\
\hline & LP & 1.85 & 2.59 & 2.20 \\
\hline \multirow{3}{*}{ Digital Filter } & $\mathrm{ACP}$ & $81.56 \pm 8.75$ & $80.53 \pm 9.30$ & $87.32 \pm 8.76$ \\
\hline & ART & $37.17 \pm 4.85$ & $26.96 \pm 3.60$ & $35.86 \pm 4.13$ \\
\hline & LP & 2.19 & 2.99 & 2.43 \\
\hline \multirow{3}{*}{ Visual Learning } & $\mathrm{ACP}$ & $91.67 \pm 7.93$ & $91.46 \pm 7.56$ & $93.54 \pm 6.06$ \\
\hline & ART & $10.83 \pm 1.39$ & $10.04 \pm 0.80$ & $10.58 \pm 3.57$ \\
\hline & LP & 8.46 & 9.11 & 8.84 \\
\hline \multirow{3}{*}{ Memory Scanning } & $\mathrm{ACP}$ & $61.88 \pm 10.91$ & $74.44 \pm 10.70$ & $66.21 \pm 8.39$ \\
\hline & ART & $25.29 \pm 4.22$ & $25.63 \pm 2.13$ & $23.63 \pm 2.9$ \\
\hline & LP & 2.45 & 2.91 & 2.80 \\
\hline \multirow{3}{*}{ Logical Events } & $\mathrm{ACP}$ & $75.42 \pm 11.40$ & $79.38 \pm 9.76$ & $87.29 \pm 8.67$ \\
\hline & ART & $60.00 \pm 11.97$ & $75.42 \pm 8.09$ & $91.63 \pm 14.12$ \\
\hline & LP & 1.26 & 1.05 & 0.95 \\
\hline \multirow{3}{*}{ Continuous Addition \& Subtraction } & $\mathrm{ACP}$ & $72.50 \pm 12.35$ & $87.15 \pm 10.05$ & $85.63 \pm 8.77$ \\
\hline & ART & $56.42 \pm 10.67$ & $53.33 \pm 4.86$ & $65.92 \pm 9.17$ \\
\hline & LP & 1.29 & 1.63 & 1.3 \\
\hline
\end{tabular}


Table A4. The final results of the ACP, ART and LP of the relevant test items on cloudy days.

\begin{tabular}{|c|c|c|c|c|}
\hline \multirow{3}{*}{ Test Item } & \multirow{3}{*}{ Indicator } & \multicolumn{3}{|c|}{ Test Conditions } \\
\hline & & \multicolumn{3}{|c|}{ Cloudy Days } \\
\hline & & Small Classroom & Medium Classroom & Large Classroom \\
\hline \multirow{4}{*}{ Letter Search } & $\mathrm{ACP}$ & $71.97 \pm 11.89$ & $77.64 \pm 13.54$ & $83.68 \pm 9.41$ \\
\hline & ART & $23.42 \pm 2.12$ & $21.67 \pm 0.76$ & $20.50 \pm 1.88$ \\
\hline & LP & 3.07 & 3.58 & 4.08 \\
\hline & $\mathrm{ACP}$ & $77.71 \pm 11.36$ & $90.49 \pm 7.55$ & $88.03 \pm 8.14$ \\
\hline \multirow{2}{*}{ Word Color Interference } & ART & $19.13 \pm 3.93$ & $15.13 \pm 1.51$ & $15.42 \pm 2.40$ \\
\hline & LP & 4.06 & 5.98 & 5.71 \\
\hline \multirow{4}{*}{ Graphic Overlay } & $\mathrm{ACP}$ & $72.86 \pm 14.76$ & $89.05 \pm 8.74$ & $86.60 \pm 9.92$ \\
\hline & ART & $24.96 \pm 2.93$ & $24.08 \pm 2.68$ & $25.42 \pm 4.79$ \\
\hline & LP & 2.92 & 3.70 & 3.41 \\
\hline & $\mathrm{ACP}$ & $63.56 \pm 11.52$ & $82.85 \pm 12.34$ & $85.07 \pm 9.71$ \\
\hline \multirow[t]{3}{*}{ Stereo Vision } & ART & $10.54 \pm 2.32$ & $7.63 \pm 0.99$ & $7.79 \pm 1.49$ \\
\hline & LP & 6.03 & 10.87 & 10.92 \\
\hline & $\mathrm{ACP}$ & $80.21 \pm 11.25$ & $88.82 \pm 8.31$ & $84.58 \pm 10.91$ \\
\hline \multirow{2}{*}{ Schulte Grid } & ART & $47.04 \pm 4.25$ & $32.46 \pm 1.93$ & $33.86 \pm 2.20$ \\
\hline & LP & 1.71 & 2.74 & 2.50 \\
\hline \multirow{3}{*}{ Digital Filter } & $\mathrm{ACP}$ & $76.81 \pm 10.67$ & $90.43 \pm 7.86$ & $87.53 \pm 8.57$ \\
\hline & ART & $30.21 \pm 2.16$ & $17.75 \pm 1.04$ & $26.37 \pm 2.00$ \\
\hline & LP & 2.54 & 5.09 & 3.32 \\
\hline \multirow{3}{*}{ Visual Learning } & $\mathrm{ACP}$ & $74.10 \pm 12.95$ & $90.42 \pm 8.01$ & $88.13 \pm 9.89$ \\
\hline & ART & $7.88 \pm 1.24$ & $8.08 \pm 0.48$ & $5.92 \pm 1.18$ \\
\hline & LP & 9.41 & 11.19 & 14.89 \\
\hline \multirow{3}{*}{ Memory Scanning } & $\mathrm{ACP}$ & $80.82 \pm 8.69$ & $83.81 \pm 8.29$ & $85.49 \pm 7.85$ \\
\hline & ART & $21.71 \pm 1.72$ & $20.79 \pm 1.33$ & $20.83 \pm 2.58$ \\
\hline & LP & 3.72 & 4.03 & 4.10 \\
\hline \multirow{3}{*}{ Logical Events } & $\mathrm{ACP}$ & $72.28 \pm 10.47$ & $77.92 \pm 11.04$ & $89.58 \pm 8.38$ \\
\hline & ART & $38.88 \pm 6.59$ & $52.46 \pm 10.83$ & $53.25 \pm 7.01$ \\
\hline & LP & 1.86 & 1.49 & 1.68 \\
\hline \multirow{3}{*}{ Continuous Addition \& Subtraction } & $\mathrm{ACP}$ & $77.96 \pm 11.42$ & $87.36 \pm 8.68$ & $86.42 \pm 9.21$ \\
\hline & ART & $49.21 \pm 6.68$ & $46.21 \pm 3.77$ & $68.08 \pm 9.31$ \\
\hline & LP & 1.58 & 1.89 & 1.27 \\
\hline
\end{tabular}

Table A5. The final results of the ACP, ART and LP of the relevant test items on sunny days.

\begin{tabular}{ccccc}
\hline & & \multicolumn{3}{c}{ Test Conditions } \\
\cline { 3 - 5 } Test Item & Indicator & & Sunny Days \\
\cline { 3 - 5 } & & Small Classroom & Medium Classroom & Large Classroom \\
\cline { 3 - 5 } Letter Search & ACP & $64.76 \pm 9.77$ & $80.67 \pm 10.40$ & $75.85 \pm 10.59$ \\
& ART & $22.00 \pm 0.72$ & $22.75 \pm 0.76$ & $20.00 \pm 1.78$ \\
Word Color Interference & LP & 2.94 & 3.55 & 3.79 \\
& ACP & $82.72 \pm 10.72$ & $89.96 \pm 8.24$ & $96.54 \pm 4.08$ \\
Graphic Overlay & ART & $13.63 \pm 1.89$ & $11.92 \pm 1.44$ & $12.71 \pm 1.41$ \\
& LP & 6.07 & 7.55 & 7.60 \\
Stereo Vision & ACP & $77.42 \pm 11.34$ & $84.58 \pm 10.72$ & $92.43 \pm 8.62$ \\
& ART & $22.38 \pm 2.74$ & $20.08 \pm 1.94$ & $22.58 \pm 3.01$ \\
& LP & 3.46 & 4.21 & 4.09 \\
& ACP & $73.15 \pm 12.89$ & $84.61 \pm 10.87$ & $90.07 \pm 9.90$ \\
& ART & $8.38 \pm 1.82$ & $6.58 \pm 0.71$ & $6.71 \pm 0.86$ \\
& LP & 8.73 & 12.85 & 13.43 \\
\hline
\end{tabular}


Table A5. Cont.

\begin{tabular}{ccccc}
\hline & & \multicolumn{3}{c}{ Test Conditions } \\
\cline { 3 - 5 } Test Item & Indicator & \multicolumn{3}{c}{ Sunny Days } \\
\cline { 3 - 5 } & & Small Classroom & Medium Classroom & Large Classroom \\
\cline { 3 - 5 } Schulte Grid & ACP & $71.83 \pm 11.77$ & $88.54 \pm 10.07$ & $96.32 \pm 5.57$ \\
& ART & $46.46 \pm 4.11$ & $32.33 \pm 3.50$ & $34.52 \pm 3.28$ \\
Digital Filter & LP & 1.55 & 2.74 & 9.79 \\
& ACP & $84.91 \pm 10.90$ & $85.63 \pm 9.31$ & $22.80 \pm 1.28$ \\
Visual Learning & ART & $26.21 \pm 2.02$ & $17.92 \pm 0.98$ & 4.16 \\
& LP & 3.24 & 4.78 & $94.17 \pm 8.03$ \\
Memory Scanning & ACP & $83.38 \pm 10.96$ & $90.75 \pm 9.24$ & $3.92 \pm 0.86$ \\
& ART & $6.08 \pm 0.94$ & $9.13 \pm 0.45$ & 24.04 \\
& LP & 13.71 & 9.95 & $81.94 \pm 8.42$ \\
Logical Events & ACP & $73.54 \pm 8.89$ & $80.97 \pm 8.38$ & $20.25 \pm 2.09$ \\
& ART & $20.88 \pm 2.39$ & $24.75 \pm 1.36$ & 4.05 \\
& LP & 3.52 & 3.27 & $87.04 \pm 8.68$ \\
& ACP & $77.01 \pm 12.83$ & $86.13 \pm 8.45$ & $62.71 \pm 15.58$ \\
& ART & $48.00 \pm 9.77$ & $45.96 \pm 9.86$ & 1.39 \\
& LP & 1.60 & 1.87 & $83.96 \pm 10.00$ \\
& ACP & $74.83 \pm 11.37$ & $88.67 \pm 8.52$ & $61.46 \pm 10.23$ \\
& ART & $52.79 \pm 11.20$ & $46.04 \pm 5.88$ & 1.37
\end{tabular}

Table A6. The final results of the P and ES of the relevant test items.

\begin{tabular}{cccc}
\hline Test Item & Indicator & P & ES \\
\hline Letter Search & ACP & 0.10 & 0.26 \\
Word Color Interference & ART & 0.08 & 0.25 \\
Graphic Overlay & ACP & 0.12 & 0.22 \\
& ART & 0.06 & 0.25 \\
Stereo Vision & ACP & 0.11 & 0.20 \\
& ART & 0.09 & 0.26 \\
Schulte Grid & ACP & 0.10 & 0.30 \\
& ART & 0.03 & 0.29 \\
Digital Filter & ACP & 0.12 & 0.21 \\
& ART & 0.14 & 0.20 \\
Visual Learning & ACP & 0.11 & 0.26 \\
& ART & 0.09 & 0.18 \\
Memory Scanning & ACP & 0.12 & 0.22 \\
Logical Events & ART & 0.03 & 0.24 \\
& ACP & 0.10 & 0.19 \\
ART & ACP & 0.08 & 0.28 \\
& ART & 0.11 & 0.27 \\
& ACP & 0.20 & 0.20 \\
\end{tabular}

\section{References}

1. Giuli, V.D.; Pos, O.D.; Carli, M.D. Indoor environmental quality and pupil perception in Italian primary schools. Build. Environ. 2012, 56, 335-345. [CrossRef]

2. Yao, R.; Li, B.; Jing, L. A theoretical adaptive model of thermal comfort-Adaptive Predicted Mean Vote (aPMV). Build. Environ. 2009, 44, 2089-2096. [CrossRef]

3. Fanger, P.O.; Toftum, J. Extension of the PMV model to non-air-conditioned buildings in warm climates. Energy Build. 2002, 34, 533-536. [CrossRef]

4. American Society of Heating, Refrigerating and Air-conditioning Engineers. ASHRAE and Thermal Environmental Conditions for Human Occupancy. ASHRAE Standard 55; American Society of Heating, Refrigerating and Air-conditioning Engineers: Atlanta, GA, USA, 2017. 
5. Ergonomics of the Thermal Environment-Analytical Determination and Interpretation of Thermal Comfort Using Calculation of the PMV and PPD Indices and Local Thermal Comfort Criteria; ISO 7730-2005; British Standards Institution: London, UK, 2005.

6. CEN. Indoor Environmental Input Parameters for Design and Assessment of Energy Performance of Buildings-Addressing Indoor Air Quality, Thermal Environment, Lighting and Acoustics; EN 16798-1; CEN: Brussels, Belgium, 2019.

7. CEN. Indoor Environmental Input Parameters for Design and Assessment of Energy Performance of Buildings Addressing Indoor Air Quality, Thermal Environment, Lighting and Acoustics (Module M1-6); EN 16798-2; CEN: Brussels, Belgium, 2019.

8. Federation of European Heating, Ventilation and Air-conditioning Associations. REHVA Guidebook NO.13: Indoor Environment and Energy Efficiency in Schools_Part 1 Principles; Federation of European Heating, Ventilation and Air-conditioning Associations: Brussels, Belgium, 2010.

9. Teli, D.; Mark, F.; Patrick, J.; James, A.B. Naturally ventilated classrooms: An assessment of existing comfort models for predicting the thermal sensation and preference of primary school children. Energy Build. 2012, 53, 166-182. [CrossRef]

10. Teli, D.; James, P.A.; Jentsch, M.F. Thermal comfort in naturally ventilated primary school classrooms. Build. Res. Inf. 2013, 41, 301-316. [CrossRef]

11. Corgnati, S.P.; Ansaldi, R.; Filippi, M. Thermal comfort in Italian classrooms under free running conditions during mid seasons: Assessment through objective and subjective approaches. Build. Environ. 2008, 44, 785-792. [CrossRef]

12. Pereira, L.D.; Raimondo, D.; Corgnati, S.P.; da Silva, M.G. Assessment of indoor air quality and thermal comfort in Portuguese secondary classrooms: Methodology and results. Build. Environ. 2014, 81, 69-80. [CrossRef]

13. Zhang, S.; Lin, Z. Extending Predicted Mean Vote using adaptive approach. Build. Environ. 2020, 171. [CrossRef]

14. Zhang, S.; Lin, Z. Extended predicted mean vote of thermal adaptations reinforced around thermal neutrality. Indoor Air 2021, 31, 1227. [CrossRef] [PubMed]

15. Zhang, S.; Cheng, Y.; Fang, Z.; Lin, Z. Improved algorithm for adaptive coefficient of adaptive Predicted Mean Vote (aPMV). Build. Environ. 2019, 163, 106318. [CrossRef]

16. Kim, J.T.; Lim, J.H.; Cho, S.H.; Yun, G.Y. Development of the adaptive PMV model for improving prediction performances. Energy Build. 2015, 98, 100-105. [CrossRef]

17. Ming, R.; Yu, W.; Zhao, X.; Liu, Y.; Li, B.; Essah, E.; Yao, R. Assessing energy saving potentials of office buildings based on adaptive thermal comfort using a tracking-based method. Energy Build. 2020, 208, 109611. [CrossRef]

18. Cho, H. The effects of summer heat on academic achievement: A cohort analysis. J. Environ. Econ. Manag. 2017, 83, 185-196. [CrossRef]

19. Wyon, D.P.; Andersen, I.B.; Lundqvist, G.R. The Effects of Moderate Heat Stress on Mental Performance. Scand. J. Work Environ Health 1979, 5, 352-361. [CrossRef]

20. Lei, P. A Positive Analysis of the Relations of Study Efficiency and Indoor Temperature: Taking Guangdong Zhanjiang Normal College Library as an example. J. Libr. Inf. Sci. Agric. 2010, 22, 147-149+152.

21. Sarbu, I.; Pacurar, C. Experimental and numerical research to assess indoor environment quality and schoolwork performance in university classrooms. Build. Environ. 2015, 93, 141-154. [CrossRef]

22. Wargocki, P.; Wyon, D.P. The Effects of Moderately Raised Classroom Temperatures and Classroom Ventilation Rate on the Performance of Schoolwork by Children (RP-1257). HVACER Res. 2011, 13, 193-220.

23. Jiang, J. Research on Classroom Thermal Environment under the Comprehensive Impact of Thermal Comfort and Learning Performance during Winter in Rural Areas of Northwest China; Xi'an University of Architecture and Technology: Xian, China, 2018.

24. Toftum, J.; Kjeldsen, B.U.; Wargocki, P.; Menå, H.R.; Hansen, E.M.; Clausen, G. Association between classroom ventilation mode and learning outcome in Danish schools. Build. Environ. 2015, 92, 494-503. [CrossRef]

25. Yan, Y.; Yan, N.; Guan, Y.; Zeng, H. Impact on brain wave rhythm and learning efficiency by color temperature of artificial light sources. J. Civ. Archit. Environ. Eng. 2012, 34, 76-79+90.

26. Gu, R.; Zhang, J.; Zhou, J.; Tong, M.S. The Baroque Music's Influence on Learning Efficiency Based on the Research of Brain Cognition. In PIERS Proceedings; IEEE: Piscataway, NJ, USA, 2014.

27. Candas, V.; Libert, J.P.; Muzet, A. Heating and cooling simulations during SWS and REM sleep in man. J. Therm. Biol. 1982, 7, 155-158. [CrossRef]

28. Bellia, L.; Alfano, F.R.D.A.; Fragliasso, F.; Palella, B.I.; Riccio, G. On the interaction between lighting and thermal comfort: An integrated approach to IEQ. Energy Build. 2021, 231, 110570. [CrossRef]

29. Code for Design of Civil Buildings (GB50352-2005); Ministry of Housing and Urban-Rural Development of the People's Republic of China: Beijing, China, 2005. (In Chinese)

30. Meteorological Information Center of China Meteorological Administration, Tsinghua Meteorological Administration and Tsinghua University. Meteorological Data Set for Building Thermal Environment Analysis of China; China Construction Industry Press: Beijing, China, 2005. (In Chinese)

31. Xu, Y.C. Study on the Factors Influencing Thermal Comfort and Learning Performance of Primary and Secondary School Students In Summer; Xi'an University of Architecture and Technology: Xian, China, 2019.

32. Xu, P.T. University Classroom Construction Standards Between Multi-Fields Horizons: A Case Study of ECNU Classroom Construction Standard; East China Normal University: Shanghai, China, 2016.

33. Chen, F. Analysis of the Educational Power of Teaching Space in Universities: Taking the University's Large-scale General Classroom as an Example. Res. Educ. Dev. 2019, 39, 79-84. 
34. Fang, Z.; Li, J.Y. A Study on University Class Size Effects. Educ. Sci. 2011, 27, 59-64.

35. International Organization for Standardization. Thermal Environments Instruments and Methods for Measuring Physical Quantities; ISO 7726; International Organization for Standardization: Geneva, Switzerland, 2012.

36. Liu, H.Q. Design Method of Energy Efficiency Residential Building in Hot-Summer and Cold-Winter Zone in China. Ph.D. Thesis, Saga University, Saga, Japan, 2017.

37. Fanger, P.O. Thermal Comfort: Analysis and Applications in Environmental Engineering; McGraw-Hill: New York, NY, USA, 1970.

38. Lei, D. Experiment Research of Effects of Clothing on Human Thermal Comfort; Chongqing University: Chongqing, China, 2012.

39. Liu, H.; Zhang, Z.; Ma, X.; Lu, W.; Li, D.; Kojima, S. Optimization Analysis of the Residential Window-to-Wall Ratio Based on Numerical Calculation of Energy Consumption in the Hot-Summer and Cold-Winter Zone of China. Sustainability 2021, 13, 6138. [CrossRef]

40. Shao, Z.F. Cognitive Psychology: Theory, Experiment and Application; Shanghai Education Press: Shanghai, China, 2006.

41. Lezak, M.D.; Howieson, D.B.; Loring, D.W. Neuropsychological Assessment; Oxford University Press: New York, NY, USA, 2006.

42. Natale, V.; Alzani, A.; Cicogna, P. Cognitive efficiency and circadian typologies: A diurnal study. Personal. Individ. Differ. 2003, 35, 1089-1105. [CrossRef]

43. Feng, W.Y. Application of NES-C3 in Test of Workers Exposed to Noise; Zhejiang University: Hangzhou, China, 2003.

44. Gu, R.; Zhang, J.; Zhou, J.; Tong, M. The Baroque music's influence on learning efficiency based on the research of eye movement. In Proceedings of the 2014 IEEE International Conference on Teaching, Assessment and Learning for Engineering (TALE), Wellington, New Zealand, 8-10 December 2014; IEEE: Piscataway, NJ, USA, 2014.

45. Ma, J.Z. A Construction Study of Working Memory Test with Reliability and Validity Analysis for Primary School Students; East China Normal University: Shanghai, China, 2011.

46. Drake, A.S. Psychometrics and normative data for the Multiple Sclerosis Functional Composite: Replacing the PASAT with the Symbol Digit Modalities Test. Mult. Scler. 2010, 16, 228-237. [CrossRef]

47. Lan, L. Mechanism and Evaluation of the Effects of Indoor Environmental Quality on Human Productivity; Shanghai Jiao Tong University: Shanghai, China, 2010.

48. Hygienic Standard for Day Lighting and Artificial Lighting for Middle and Elementary School (GB7793-2010); Ministry of Health of the People's Republic of China, China National Standardization Administration Committee: Beijing, China, 2010. (In Chinese)

49. Geng, Y.; Ji, W.; Lin, B.; Zhu, Y. The impact of thermal environment on occupant IEQ perception and productivity. Build. Environ. 2017, 121, 158-167. [CrossRef]

50. Hu, Z.J.; Dai, H.Q. The comparison for assessing methods of the effect size and statistical power of ANOVA. Psychol. Explor. 2011, 31, 254-259.

51. Jacob, C. Statistical Power Analysis for the Behavioral Sciences; Taylor and Francis: Oxford, UK, 2013.

52. Cui, W.; Cao, G.; Ouyang, Q.; Zhu, Y. Influence of dynamic environment with different airflows on human performance. Build. Environ. 2013, 62, 124-132. [CrossRef]

53. Lan, L.; Lian, Z. Use of neurobehavioral tests to evaluate the effects of indoor environment quality on productivity. Build. Environ. 2009, 44, 2208-2217. [CrossRef]

54. Lan, L.; Lian, Z.; Pan, L.; Ye, Q. Neurobehavioral approach for evaluation of office workers' productivity: The effects of room temperature. Build. Environ. 2008, 44, 1578-1588. [CrossRef] 